\title{
Estimates of Gradients in Radar Moments Using a Linear Least Squares Derivative Technique
}

\author{
Matthew C. Mahalik, Brandon R. Smith, Kimberly L. Elmore, Darrel M. Kingfield, ${ }^{\mathrm{a}}$ \\ KiEl L. ORTEGA, AND TRAVIS M. SMith \\ Cooperative Institute for Mesoscale Meteorological Studies, University of Oklahoma, and NOAA/OAR National Severe Storms \\ Laboratory, Norman, Oklahoma
}

(Manuscript received 6 June 2018, in final form 27 January 2019)

\begin{abstract}
The local, linear, least squares derivative (LLSD) approach to radar analysis is a method of quantifying gradients in radar data by fitting a least squares plane to a neighborhood of range bins and finding its slope. When applied to radial velocity fields, for example, LLSD yields part of the azimuthal (rotational) and radial (divergent) components of horizontal shear, which, under certain geometric assumptions, estimate one-half of the two-dimensional vertical vorticity and horizontal divergence equations, respectively. Recent advances in computational capacity as well as increased usage of LLSD products by the meteorological community have motivated an overhaul of the LLSD methodology's application to radar data. This paper documents the mathematical foundation of the updated LLSD approach, including a complete derivation of its equation set, discussion of its limitations, and considerations for other types of implementation. In addition, updated azimuthal shear calculations are validated against theoretical vorticity using simulated circulations. Applications to nontraditional radar data and new applications to nonvelocity radar data including reflectivity at horizontal polarization, spectrum width, and polarimetric moments are also explored. These LLSD gradient calculations may be leveraged to identify and interrogate a wide variety of severe weather phenomena, either directly by operational forecasters or indirectly as part of future automated algorithms.
\end{abstract}

\section{Introduction}

Weather radars are crucial to identifying and understanding weather phenomena. Signatures of precipitation type and rate, airmass boundaries, and storm rotation are often identifiable by the size, shape, and location of gradients within specific radar fields. In reflectivity at horizontal polarization $Z_{H}$ alone, the hook echo of a supercell (Fujita 1973; Markowski 2002), a bow-shaped $Z_{H}$ maximum common to damaging wind events (Przybylinski 1995), and the manifestation of tornadic debris in the form of a "debris ball" (Burgess et al. 2002) can all be thought of as gradients. Likewise, gradient patterns such as differential reflectivity $Z_{\mathrm{DR}}$ arcs (Kumjian and Ryzhkov

\footnotetext{
${ }^{\text {a }}$ Current affiliation: Cooperative Institute for Research in Environmental Sciences, University of Colorado, and NOAA/OAR/ ESRL/Global Systems Division, Boulder, Colorado.
}

Corresponding author: Matthew Mahalik, matthew.mahalik@ noaa.gov
2008) and tornado debris signatures (TDS; Ryzhkov et al. 2005) within polarimetric data fields such as $Z_{\mathrm{DR}}$ and cross-correlation coefficient $\rho_{\mathrm{hv}}$ aid in the detection and tracking of tornadoes and diagnostics of updraft characteristics.

Radial velocity $V_{r}$ gradients have been the subject of perhaps the most study with respect to severe storms. Among a wide range of applications, $V_{r}$ gradients are used to help identify convergence along boundaries, which may be either the result of or catalyst for convection (Carbone et al. 1990; Wilson et al. 1992), midlevel convergence within a thunderstorm that can signal the onset of damaging winds (Schmocker et al. 1996), divergent flows from downdrafts impinging on the surface or storm exhaust at the cloud top (Roberts and Wilson 1989; Smith et al. 2004), and rotation associated with mesocyclones (Donaldson 1970) and tornadoes (Donaldson 1978; Brown et al. 1978). More recently, relationships between the magnitudes of $V_{r}$ gradients in rotation signatures (measured as velocity difference, rotational velocity, etc.) and tornado occurrence, intensity, and damage probability, have been 
derived (Alexander and Wurman 2008; Smith et al. 2015; Thompson et al. 2017).

Typically, the relative magnitude of rotation in severe local storms is quantified as the magnitude of the difference between the local maxima in inbound and outbound $V_{r}$. This "peak-to-peak" technique yields a quantity often referred to as "delta velocity," which may be normalized by the distance between the $V_{r}$ extrema (Mitchell et al. 1998; Stumpf et al. 1998). This one-dimensional approach is effective in estimating the relative, local rate of rotation, but it excludes surrounding data points, which may include valuable information about the rotating feature beyond simply the maximum rotation rate at its core. Other limiting assumptions of the peak-to-peak approach include the following: 1 ) the center of the circulation lies exactly between the extrema, which may not be possible to determine with coarse beamwidths and physical radar sampling limitations (Burgess et al. 1993; Wood and Brown 1997); 2) the extrema themselves are accurate measurements, which is not always true due to noisy or poorly dealiased data (Zittel et al. 2001); and 3) peak-topeak delta velocity will usually be biased low unless the tornado is very wide, due to azimuthal smearing and averaging of the radar beam over a relatively narrow wind max (Wood and Brown 2011).

In response to the need for an alternative approach to quantifying $V_{r}$ gradients in a mathematically robust but sufficiently straightforward way for real-time computation and operational use, Elmore et al. (1994) and Smith and Elmore (2004, hereafter SE04) introduced the linear, least squares derivative (LLSD) approach to radar data analysis. LLSD is a statistical data analysis approach that quantifies the gradient of a scalar field as a two-dimensional, least squares plane that is fit to a local neighborhood (or "kernel") of $V_{r}$ data or other radar measurements. The slope of this best-fit plane is the local gradient at the center of the kernel. This approach includes local information about the entire feature by using surrounding data and effectively smoothing extreme values to minimize the effect of outliers caused by poor data quality. The total gradient is decomposed into azimuthal and radial components, which are calculated simultaneously for any given radar scan. When applied to $V_{r}$ fields, the across-azimuth gradient represents the rotational shear component, referred to as "AzShear" (azimuthal shear; Miller et al. 2013). The complementary, along-azimuth $V_{r}$ gradient yields the divergent shear component, or "DivShear." Typical AzShear and DivShear fields for a tornadic supercell are shown in Fig. 1.

Although $V_{r}$ is a vector directed along the radial, treating it as a local scalar provides numerous advantages. For example, scalar LLSD gradients can be easily gridded for research or operational use. At the time of this writing, gridded AzShear is an operational product within the Multi-Radar Multi-Sensor (MRMS) system (Smith et al. 2016). In recent years, the application of AzShear in a wide range of operational and research initiatives (Heinselman et al. 2008, 2012; Stumpf et al. 2012; Davis and Parker 2014; Snyder and Ryzhkov 2015; Skinner et al. 2016; Dawson et al. 2017; Stough et al. 2017, among others), coupled with an increase in computational capacity, has motivated attempts to improve the accuracy of LLSD-derived products. For example, Miller et al. (2013) and Newman et al. (2013) described postprocessing methods to overcome general limitations of the LLSD technique by improving the ability of AzShear to identify rotation at the storm and tornado scales through the use of multiple hypothesis tracking (a technique used to isolate continuous tracks of rotating objects) and empirically derived, range-based shear corrections, respectively.

Unlike postprocessing approaches, the work presented here focuses directly on the underlying LLSD equations and specific improvements upon the original SE04 methodology and provides a technique generalized for calculating LLSD gradients of any data field. The LLSD equations were rederived without regard to the simplifying assumptions discussed in SE04, which were at the time necessitated by computational limitations. Updated LLSD outputs of AzShear were then validated using a suite of simulated Rankine vortices with known rotation attributes (size, strength, and range from radar). In addition, the LLSD equations were applied to a variety of radar fields to investigate the method's utility in a range of meteorological phenomena, producing gradient fields beyond only AzShear for both $V_{r}$ and non- $V_{r}$ radar variables.

The effectiveness of direct use of LLSD gradients by forecasters is dependent on the application. For example, while time-accumulated AzShear (often referred to as "rotation tracks"; Miller et al. 2013) has been demonstrated as a valuable tool for relaying tornado information to end users (Kuster et al. 2017), gradient fields of $\rho_{\mathrm{hv}}$ may be less intuitive. In essence, all LLSD gradients are quantitative representations of signatures that human forecasters are trained to visually identify but that can sometimes be difficult to quantify. As a result, many of the examples presented here show more promise as an intermediate product toward future featureidentification or interrogation algorithm development.

In this paper, the mathematical derivation of the complete set of LLSD equations is shown in section 2 . Section 3 discusses the mathematic and geometric considerations of the LLSD kernel, and section 4 provides examples of LLSD applications to various radar data. The LLSD fields shown throughout were produced using the National Severe Storms Laboratory (NSSL) 


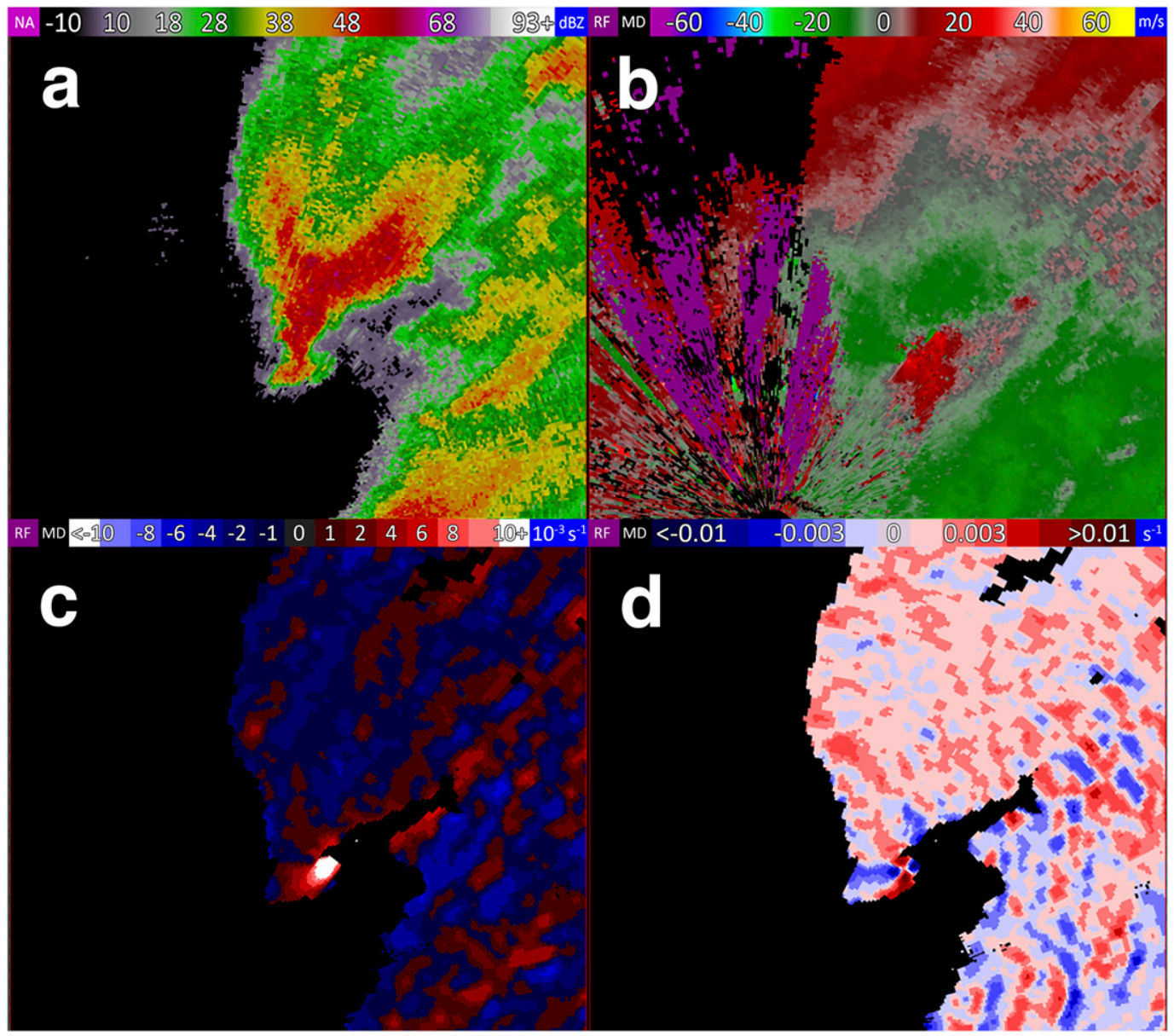

FIG. 1. Single-radar (a) $0.5^{\circ} \mathrm{QC} Z_{H}$, (b) $0.5^{\circ}$ dealiased $V_{r}$, (c) $0.5^{\circ} \mathrm{AzShear}$, and (d) $0.5^{\circ}$ DivShear depiction of a tornadic supercell from the Cheyenne, WY (KCYS), radar at 2231 UTC 12 Jun 2017. The low-level mesocyclone is visible in (c) as a maximum in AzShear (white) and in (d) as coupled extrema of positive and negative DivShear (deep red and blue, respectively). AzShear and DivShear are thresholded by $>20 \mathrm{dBZ} 0.5^{\circ} \mathrm{QC} Z_{H}$.

Warning Decision Support System-Integrated Information (WDSS-II; Lakshmanan et al. 2007b) software suite. Many of the LLSD gradient fields are thresholded by quality-controlled (QC) $Z_{H}$ (Lakshmanan et al. 2007a) to focus solely on storm processes.

\section{Derivation}

Before LLSD gradients are calculated for any input field, the data are passed through a median filter (Huang et al. 1979) to reduce noise, fill in potential missing data, and filter outliers. Any input $V_{r}$ data should be dealiased beforehand. The filter used by NSSL LLSD algorithms measures three azimuths across by three range gates deep and assigns the median value of those nine data points to the center gate of the neighborhood if at least five of the eight surrounding gates contain valid data. Measurements within the $3 \times 3$ neighborhood are assumed to occur at regular radius and azimuth intervals.
This filter is applied to each individual data point within the field. Any gates with missing or range-folded data remaining in its kernel are discarded. The resulting filtered data are then passed to the LLSD equations.

The original LLSD equations introduced by Elmore et al. (1994) and expanded by SE04 were simplified at the time for computational efficiency. In particular, it was assumed that the data within the LLSD kernel are locally Cartesian and symmetric about the kernel center. As such, these calculations implemented a locally constant azimuthal spacing between data points. This assumption becomes especially problematic in two situations. First, near the radar, the inclusion of additional, azimuthally narrow gates may cause the LLSD kernel to wrap around the radar such that the straightline distance across the kernel may be smaller than the polar-coordinate distance within the kernel. As a result, the gradient would be calculated across a smaller distance, producing artificially high shears surrounding the 


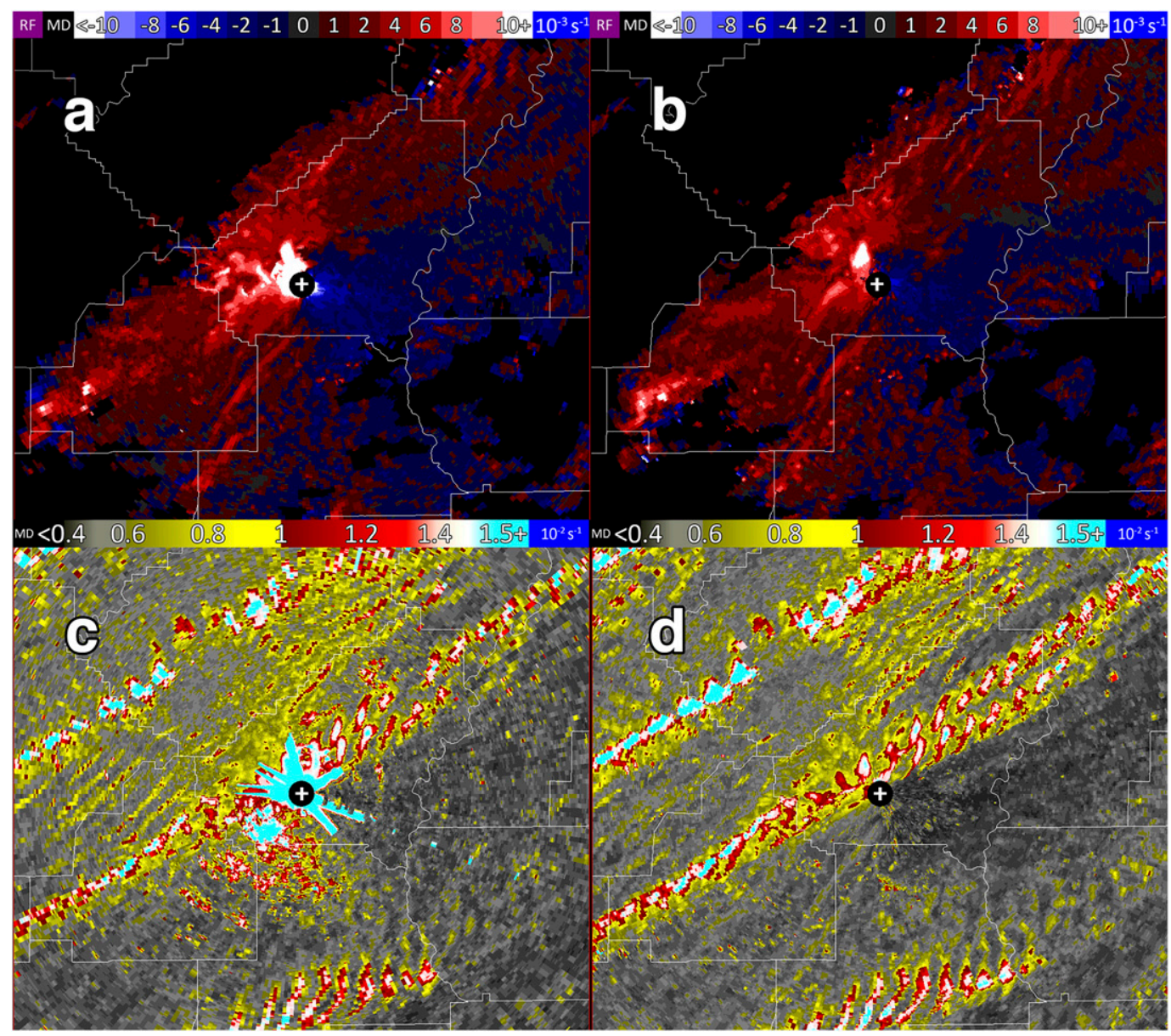

FIG. 2. Single-radar 0-2 km AGL layer-maximum AzShear for a tornadic supercell near the Birmingham, AL (KBMX), radar (denoted by the white cross) at 0009 UTC 28 Apr 2011 calculated using (a) legacy LLSD equations and (b) the updated LLSD algorithm, and single-radar 0-2 km AGL rotation tracks for the 12-h period ending 0300 UTC 28 Apr 2011 using (c) legacy and (d) updated LLSD equations. The vortex path is more easily identifiable in both the instantaneous AzShear and accumulated tracks using the updated LLSD algorithm in (b) and (d), where artificially high AzShear is not present.

radar location. Second, if the LLSD kernel covers a large distance radially (as in along-radial gradient applications), the Cartesian assumption may be violated because the radar beam physically broadens with range, also decreasing the across-beam resolution with distance from the radar. If the change in azimuthal spacing is sufficiently large, the gradient would be calculated over a larger horizontal distance, resulting in artificially low AzShear.

Given recent increases in processing capabilities, an updated LLSD methodology was developed from the full derivation below, which is not subject to the same restrictions. While the updated equations greatly alleviate these problems (section 3), the method is not perfect at very large or small distances from the radar due to sampling limitations, and similar but less-severe artifacts may be present in some situations.
Since LLSD calculates a best-fit plane for areas across several data points, we use a general, linear, least squares approximation, which yields the largest rate of change in any direction. This estimate is derived from the minimization of a general, first-order, planar equation as in Eq. (1):

$$
\mu(b, c)=a_{0}+a_{1}(b)+a_{2}(c),
$$

where $\mu(b, c)$ is the linear fit of the coefficient vector $\mathbf{u}=\left[\begin{array}{lll}a_{0} & a_{1} & a_{2}\end{array}\right]$, modified by coefficients $b$ and $c$. For meteorological purposes, the three components of $\mathbf{u}$ include a constant $u_{0}$, and the radial $u_{r}$ and azimuthal $u_{\theta}$ derivatives (shears) of a field [Eq. (2)]:

$$
\mathbf{u}=\left[u_{0} u_{r} u_{\theta}\right] .
$$

This shear vector can be found by minimizing the sum of squares over $n$ data points. This sum of squares $R$ 
may be locally weighted by weight $w_{i}$, within the LLSD kernel [Eq. (3)]:

$$
R \equiv \sum_{i=1}^{n} w_{i}\left[Y_{i}-\mu(b, c)\right]^{2} .
$$

The linear fit is a function of the response variable $Y_{i}$. For radar data, $Y_{i}$ is a two-dimensional radar variable $u_{i j}$, which is itself dependent on the offset, in range $\Delta r_{i j}$ and azimuth $\Delta \theta_{i j}$, from the kernel center $(r=0, \theta=0)$, where $\Delta \theta_{i j}\left(\Delta r_{i j}\right)$ is a distance in the azimuthal (radial) direction. If we define the kernel dimensions as $m \times n$ such that index $i(j)$ runs from $i=0$ to $i=m$ (from $j=0$ to $j=n$ ), then $R$ can be calculated over each point $k=(i, j)$ within the kernel. A discussion of kernel size considerations is presented in section 3. Then, $\mu$ [Eq. (1)] and $R$ [Eq. (3)] can be reformulated as in Eqs. (4) and (5):

$\mu(r, \theta)=u(r, \theta)=u_{0}+u_{r} \Delta r_{k}+u_{\theta} \Delta \theta_{k}, \quad$ and

$R=\sum_{k=0}^{m \times n} w_{k}\left[u\left(r_{k}, \Delta \theta_{k}\right)-\left(u_{0}+u_{r} \Delta r_{k}+u_{\theta} \Delta \theta_{k}\right)\right]^{2}$.

As dictated by the least squares technique, $R$ is minimized by setting its derivative to zero in each direction. This yields the following normal, linear, least squares derivatives [Eqs. (6a)-(6c)]:

$$
\begin{aligned}
& \frac{\partial R}{\partial u_{r}}=0=\sum_{k=0}^{m \times n} 2\left(-u_{k}+u_{0}+u_{r} \Delta r_{k}+u_{\theta} \Delta \theta_{k}\right) w_{k} \Delta r_{k}, \quad \text { (6a) } \\
& \frac{\partial R}{\partial u_{\theta}}=0=\sum_{k=0}^{m \times n} 2\left(-u_{k}+u_{0}+u_{r} \Delta r_{k}+u_{\theta} \Delta \theta_{k}\right) w_{k} \Delta \theta_{k}, \quad \text { and }
\end{aligned}
$$

$\frac{\partial R}{\partial u_{0}}=0=\sum_{k=0}^{m \times n} 2\left(-u_{k}+u_{0}+u_{r} \Delta r_{k}+u_{\theta} \theta_{k}\right) w_{k}$.

Terms in these equations are combined and rearranged to produce the complete system of least squares derivatives [Eqs. (7a)-(7c)]:

$$
\begin{aligned}
\sum_{k=0}^{m \times n} u_{k} w_{k} \Delta r_{k}= & \sum_{k=0}^{m \times n} u_{0} w_{k} \Delta r_{k}+\sum_{k=0}^{m \times n} u_{r} w_{k}\left(\Delta r_{k}\right)^{2} \\
& +\sum_{k=0}^{m \times n} u_{\theta} w_{k} \Delta r_{k} \Delta \theta_{k}, \\
\sum_{k=0}^{m \times n} u_{k} w_{k} \Delta \theta_{k}= & \sum_{k=0}^{m \times n} u_{0} w_{k} \Delta \theta_{k}+\sum_{k=0}^{m \times n} u_{r} w_{k} \Delta r_{k} \Delta \theta_{k} \\
& +\sum_{k=0}^{m \times n} u_{\theta} w_{k}\left(\Delta \theta_{k}\right)^{2}, \quad \text { and }
\end{aligned}
$$

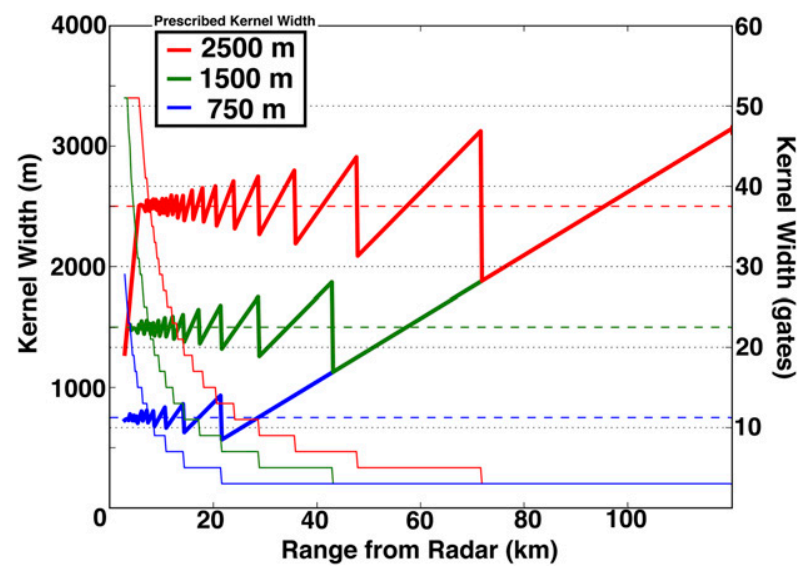

FIG. 3. Summary of the variability of LLSD kernel size with range for prescribed azimuthal widths of 750 (blue), 1500 (green), and $2500 \mathrm{~m}$ (red) for $0.5^{\circ}$ superresolution radar data from a WSR$88 \mathrm{D}$. The actual width of the kernel $(\mathrm{m})$ is shown as the thick line, while the thin lines show the respective number of gates in the kernel. The prescribed kernel widths $(\mathrm{m})$ are provided for reference as horizontal dashed lines. Values for any kernel size converge at some range; here, all three prescribed kernel sizes result in the same true kernel size beyond a range of approximately $72 \mathrm{~km}$.

$$
\begin{aligned}
\sum_{k=0}^{m \times n} u_{k} w_{k}= & \sum_{k=0}^{m \times n} u_{0} w_{k}+\sum_{k=0}^{m \times n} u_{r} w_{k} \Delta r_{k} \\
& +\sum_{k=0}^{m \times n} u_{\theta} w_{k} \Delta \theta_{k} .
\end{aligned}
$$

Here the derivations deviate from SE04, which assumed perfectly symmetrical data about the kernel center and neglected all terms containing off-diagonal coefficients. In this derivation, all terms are preserved.

From Eqs. (7a)-(7c), the individual shear components in Eq. (4) can be found. Equations (7a)-(7c) can be expressed in matrix form as $\mathbf{M X}=\mathbf{Y}$, where $\mathbf{M}$ is a matrix of the position $\left(\Delta r_{k}\right.$ and $\left.\Delta \theta_{k}\right)$ and weighting $\left(w_{k}\right)$ coefficients, and $\mathbf{X}$ is a matrix of shear components [Eq. (8)], where all summations remain from $k=0$ to $k=m \times n$ :

$$
\begin{aligned}
& {\left[\begin{array}{ccc}
\Sigma w_{k} \Delta r_{k} \Delta \theta_{k} & \Sigma w_{k} \Delta \theta_{k}^{2} & \Sigma w_{k} \Delta \theta_{k} \\
\Sigma w_{k} \Delta r_{k}^{2} & \Sigma w_{k} \Delta r_{k} \Delta \theta_{k} & \Sigma w_{k} \Delta r_{k} \\
\Sigma w_{k} \Delta r_{k} & \Sigma w_{k} \Delta \theta_{k} & \Sigma w_{k}
\end{array}\right]\left[\begin{array}{l}
u_{\theta} \\
u_{r} \\
u_{0}
\end{array}\right]} \\
& =\left[\begin{array}{c}
\Sigma w_{k} \Delta r_{k} u_{k} \\
\Sigma w_{k} \Delta \theta_{k} u_{k} \\
\Sigma w_{k} u_{k}
\end{array}\right] .
\end{aligned}
$$

The individual derivatives are found by multiplying the equation by the inverse of the coefficient matrix [Eq. (9)]:

$$
\mathbf{X}=\mathbf{M}^{-1} \mathbf{Y}
$$



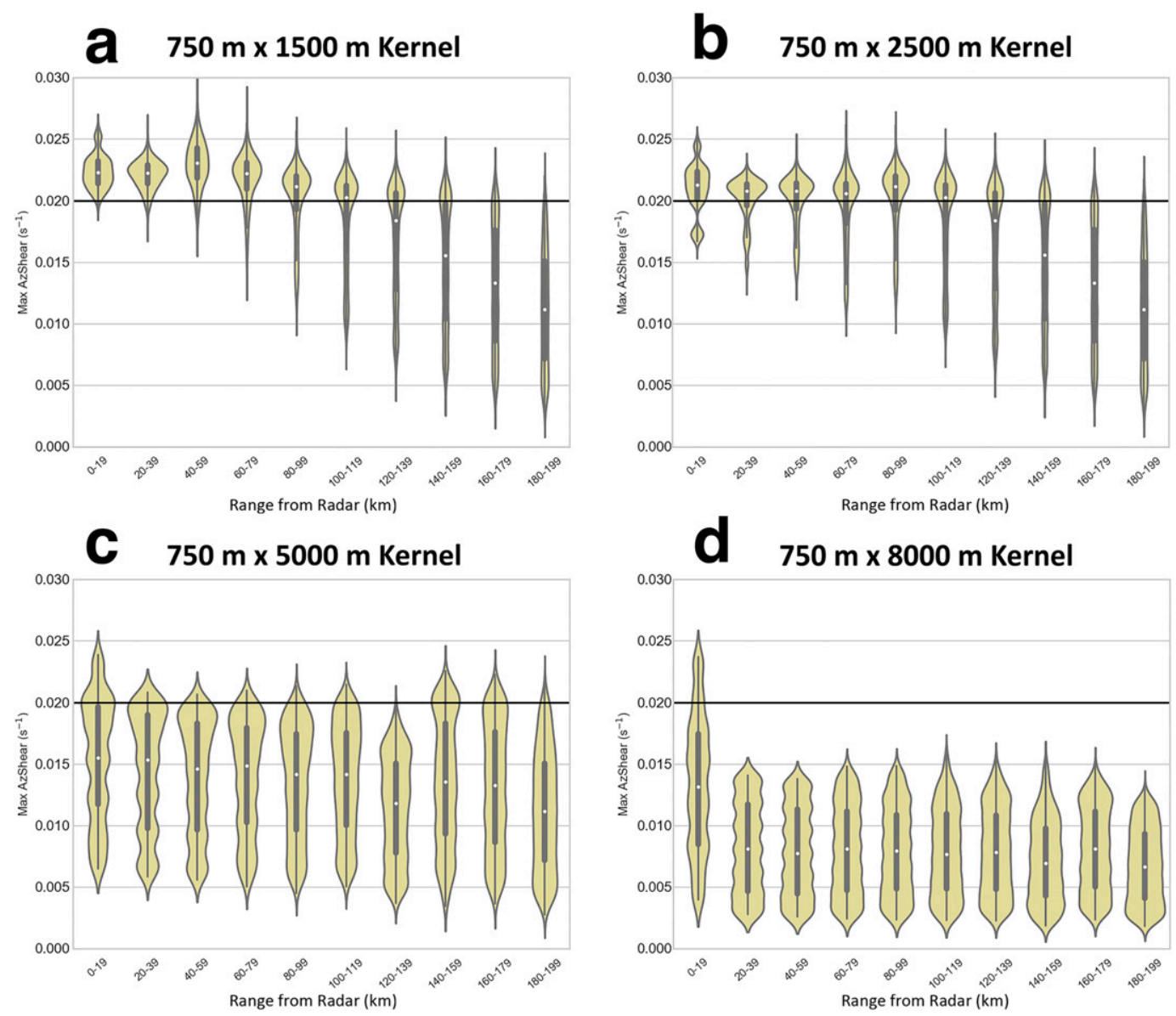

FIG. 4. Violin plots showing the distribution of maximum AzShear values for a suite of vortices simulated by a Rankine combined vortex model with theoretical half- $\zeta=0.02 \mathrm{~s}^{-1}$ for LLSD kernels with target azimuthal widths of (a) 1500, (b) 2500, (c) 5000, and (d) $8000 \mathrm{~m}$.

The inverse of matrix $\mathbf{M}$ can be calculated using the determinant $D$ and adjugate of matrix $\mathbf{M}[\operatorname{adj}(\mathbf{M})]$ under the condition $D \neq 0$ [Eq. (10)]. The individual terms of the adjugate matrix are written as $a_{i j}$ :

$$
\mathbf{M}^{-1}=\frac{1}{D} \operatorname{adj}(\mathbf{M})=\left[\begin{array}{lll}
a_{11} & a_{12} & a_{13} \\
a_{21} & a_{22} & a_{32} \\
a_{31} & a_{23} & a_{33}
\end{array}\right]\left(\frac{1}{D}\right) .
$$

This allows Eq. (9) to be expressed as Eq. (11), which can then be solved for both shear components, yielding the final LLSD horizontal shear equations for the azimuthal [Eq. (12a)] and divergent [Eq. (12b)] components:

$$
\left[\begin{array}{c}
u_{\theta} \\
u_{r} \\
u_{0}
\end{array}\right]=\left[\begin{array}{lll}
a_{11} / D & a_{12} / D & a_{13} / D \\
a_{21} / D & a_{22} / D & a_{32} / D \\
a_{31} / D & a_{23} / D & a_{33} / D
\end{array}\right]\left[\begin{array}{c}
\Sigma w_{k} \Delta r_{k} u_{k} \\
\Sigma w_{k} \Delta \theta_{k} u_{k} \\
\Sigma w_{k} u_{k}
\end{array}\right]
$$

$$
\begin{aligned}
u_{\theta}= & \sum_{k=0}^{m \times n} w_{k} \Delta r_{k} u_{k}\left(\frac{a_{11}}{D}\right)+\sum_{k=0}^{m \times n} w_{k} \Delta \theta_{k} u_{k}\left(\frac{a_{12}}{D}\right) \\
& +\sum_{k=0}^{m \times n} w_{k} u_{k}\left(\frac{a_{13}}{D}\right), \text { and } \\
u_{r}= & \sum_{k=0}^{m \times n} w_{k} \Delta r_{k} u_{k}\left(\frac{a_{21}}{D}\right)+\sum_{k=0}^{m \times n} w_{k} \Delta \theta_{k} u_{k}\left(\frac{a_{22}}{D}\right) \\
& +\sum_{k=0}^{m \times n} w_{k} u_{k}\left(\frac{a_{23}}{D}\right) .
\end{aligned}
$$

The fully expanded forms of Eqs. (12a) and (12b) and list of variables for each are found in appendixes A and B, respectively. Some of the variables in these equations can be adjusted to suit specific research needs. Several of the aspects discussed in section 3 are in the context of the current, operational MRMS configuration, though all are important considerations for any application of the LLSD technique. 

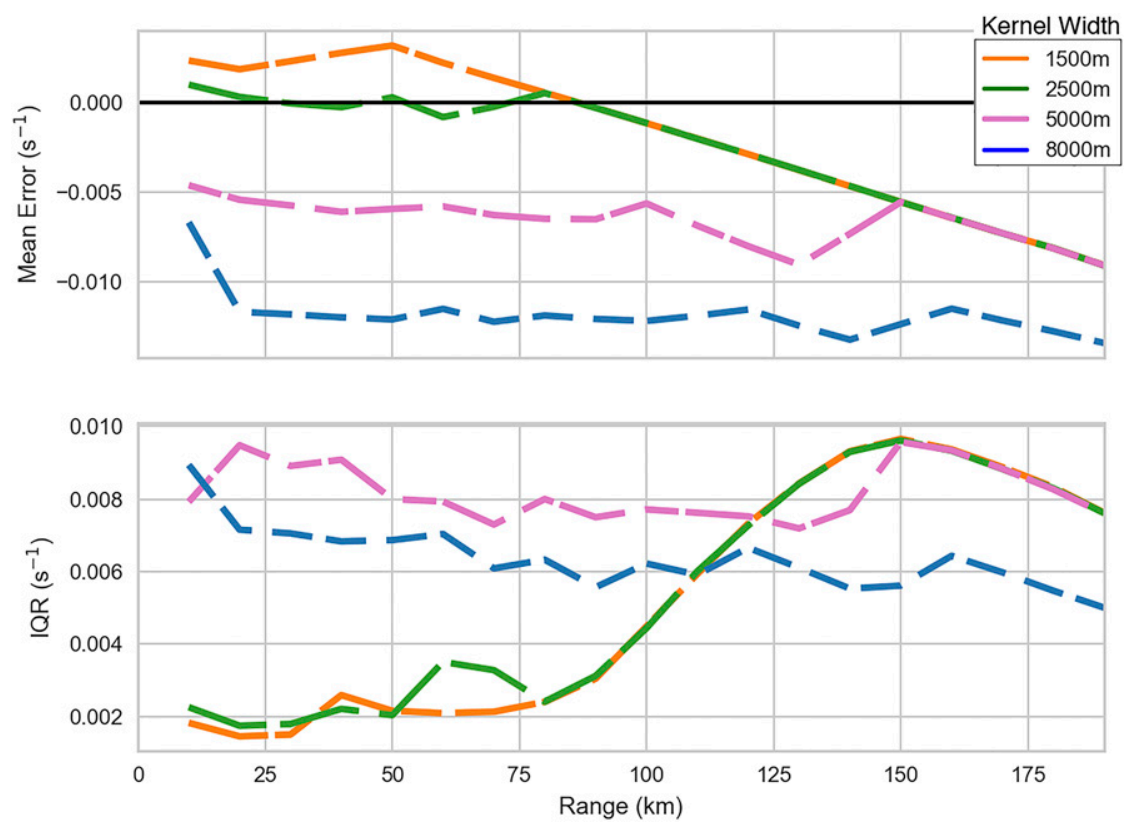

FIG. 5. (top) Average error (calculated as the mean of all differences between calculated AzShear and theoretical AzShear) and (bottom) average median-centered IQR (the difference between the 75th and 2fifth percentile values) of all simulated vortices in Fig. 4 with theoretical half- $\zeta=0.02 \mathrm{~s}^{-1}$ for LLSD kernels with target azimuthal widths of $1500,2500,5000 \mathrm{~m}$, and $8000 \mathrm{~m}$. At range $<100 \mathrm{~km}$, smaller kernels tend to have lower mean errors and lower IQR, suggesting more consistency and accuracy, and less sensitivity to circulation geometry.

\section{Discussion}

Much of the immediate utility of the LLSD equation adjustments can be seen in two-dimensional gradient fields of radar data, as in Figs. 1c and 1d. LLSD gradients are calculated for and assigned to each individual gate occupied by valid input radar data. They are pieced together onto the same grid as the input field, resulting in complete gradient fields that are used to visualize gradients within the context of an entire radar scan. Internal testing of LLSD-derived products has shown an improvement of AzShear and other LLSD gradient calculations using the updated LLSD equations over legacy equations. For example, much of the artificially high AzShear within $5-10 \mathrm{~km}$ of the radar, commonly observed with legacy LLSD equations, is mitigated using the updated LLSD algorithm, allowing vortices very near the radar to be more easily distinguished (Fig. 2).

\section{a. Kernel size considerations}

All LLSD gradient calculations are highly sensitive to the size of the LLSD kernel. For example, consider the AzShear calculation for a hypothetical, symmetrical, isolated vortex in the center of the kernel. Assuming the entire vortex fits within the kernel, a large kernel will include more low-shear gates away from the vortex core than a smaller one, thereby reducing the overall shear within the kernel. Conversely, a small kernel is susceptible to missing gates that lie on the outer edge of a circulation, thus slightly overestimating the maximum rotation of the vortex as a whole.

The LLSD algorithm uses a constant, predefined kernel size (in meters). It may be manually adjusted to best suit the type of analysis being performed but is constant across the volume during processing. For example, AzShear uses a kernel with a prescribed azimuthal width of $2500 \mathrm{~m}$ and radial depth of $750 \mathrm{~m}$, and DivShear uses kernel dimensions of $750-\mathrm{m}$ azimuthal width by $1500-\mathrm{m}$ radial depth. In general, the azimuthal dimension of a kernel used to calculate across-azimuth gradients should be at least as large as its radial dimension, and vice versa.

Because of the physical limitations of radar sampling, azimuthal spacing increases with range. To maintain an approximately constant physical kernel width, the number of azimuthal gates within the kernel decreases with range to compensate for increasing gate widths. Thus, at small ranges from the radar, the kernel contains a greater number of radials than a kernel located at a larger range. At each range, the number of radials required to construct the kernel is explicitly calculated, meaning that the kernel size updates as frequently as geometrically possible to maintain an accurate width. This adaptive 


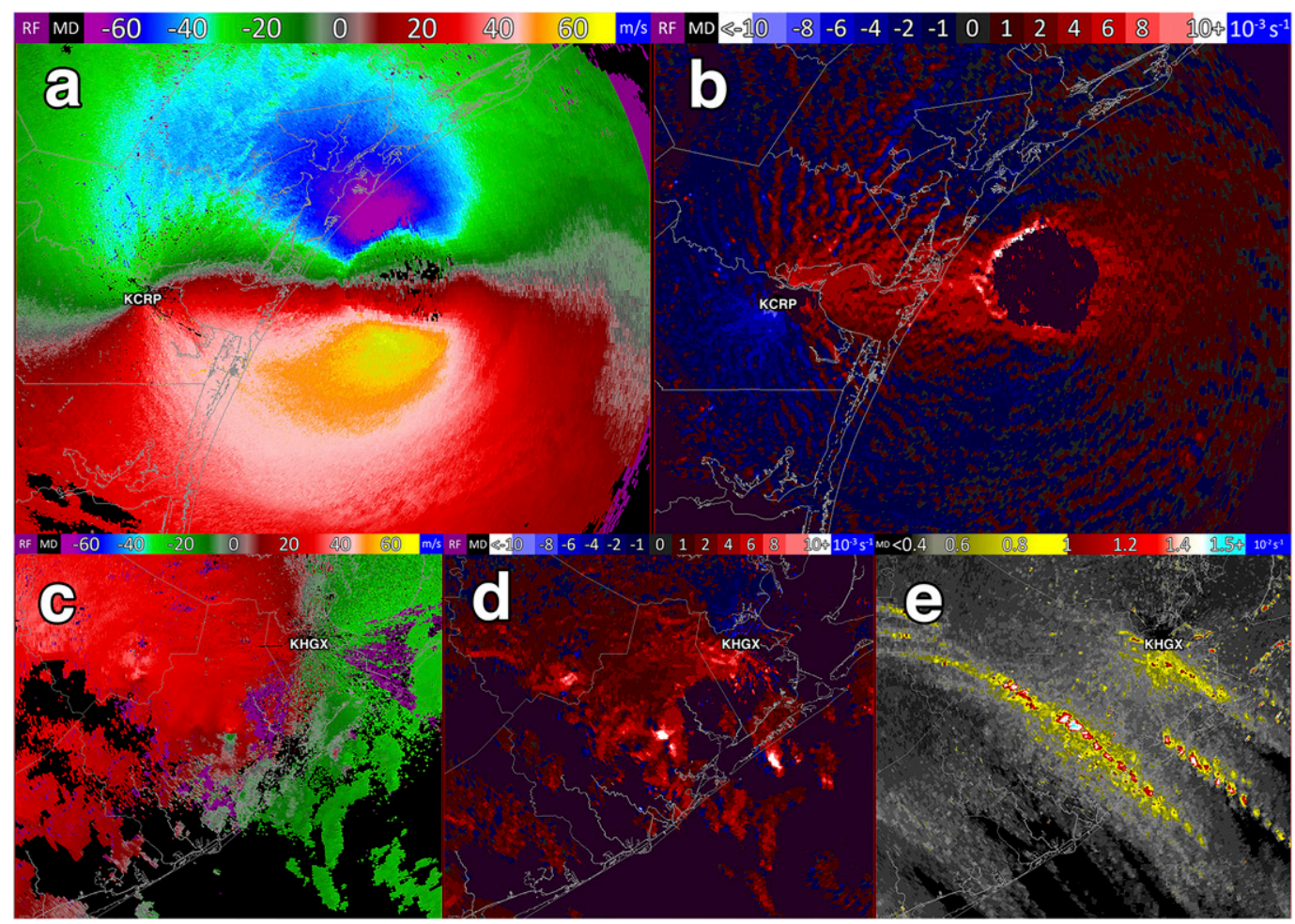

FIG. 6. Single-radar observations during Hurricane Harvey on 26 Aug 2017: (a) $0.5^{\circ}$ dealiased $V_{r}$ and (b) corresponding 0-2 km AGL layer-maximum AzShear from the Corpus Christi, TX (KCRP), radar near the center of Hurricane Harvey at 0039 UTC, shortly before landfall; (c) dealiased $0.5^{\circ}$ velocity associated with outer rainband cells from the Houston/Galveston, TX (KHGX), radar at 0244 UTC, (d) corresponding 0-2 km AGL layer-maximum AzShear, and (e) 2-h accumulated 0-2 km AGL layer-maximum rotation tracks ending at 0252 UTC. In (b), AzShear highlights the strong gradient between major hurricane-force winds in the eyewall and weak winds in the eye. Low-level circulations, some tornadic, are tracked using AzShear in (d) and (e). The AzShear fields were calculated using the same default LLSD kernel size.

approach is not perfect and results in 1) inaccuracies at very large or very small distances from the radar and 2) discontinuities at certain ranges in between (Fig. 3). Near the radar, where azimuthal spacing is smallest, the kernel may wrap sufficiently around the radar to violate the assumption that the kernel columns are practically parallel. As a result, an upper limit on the number of radials in a single kernel is imposed. When applying a kernel with an azimuthal width of $2500 \mathrm{~m}$ to a $0.5^{\circ}$ elevation scan of superresolution WSR-88D radar data, this limit is 51 radials. This threshold was selected through subjective evaluation of the AzShear product and affects kernels centered at a range of $6 \mathrm{~km}$ or less.

The LLSD equations mathematically require a minimum of three radials and three range gates within a kernel to calculate the $2 \mathrm{D}$ gradient. As the kernel expands laterally with increasing azimuthal spacing, the number of radials that fit inside the kernel will eventually reach this minimum. The range at which this occurs is dependent on radar properties and the prescribed kernel size. At all ranges beyond this point, the prescribed kernel width is no longer maintained. For an AzShear kernel with an azimuthal width of $2500 \mathrm{~m}$ applied to a WSR-88D $0.5^{\circ}$ elevation scan, this occurs at ranges greater than approximately $72 \mathrm{~km}$. Beyond this range, the kernel width exceeds the prescribed width, meaning that the derivatives are taken over a larger area, creating a generally lower gradient estimate (Newman et al. 2013). This is visually manifested as a "smearing" effect across radials at large ranges, which is a common artifact of radar data. The default DivShear configuration's kernel, which is narrower in azimuth, is subject to the same effect, though the smearing problem begins closer to the radar than with AzShear.

\section{b. Kernel weighting}

By default, LLSD products use a uniform weight for each gate within the kernel, such that $w_{k}=1$ for all $k$ in Eqs. (12a) and (12b) (Miller et al. 2013). Another option is to employ a nonuniform weighting scheme, such as Cressman weighting (Cressman 1959), so that gates farther from the center of the kernel receive less weight. 


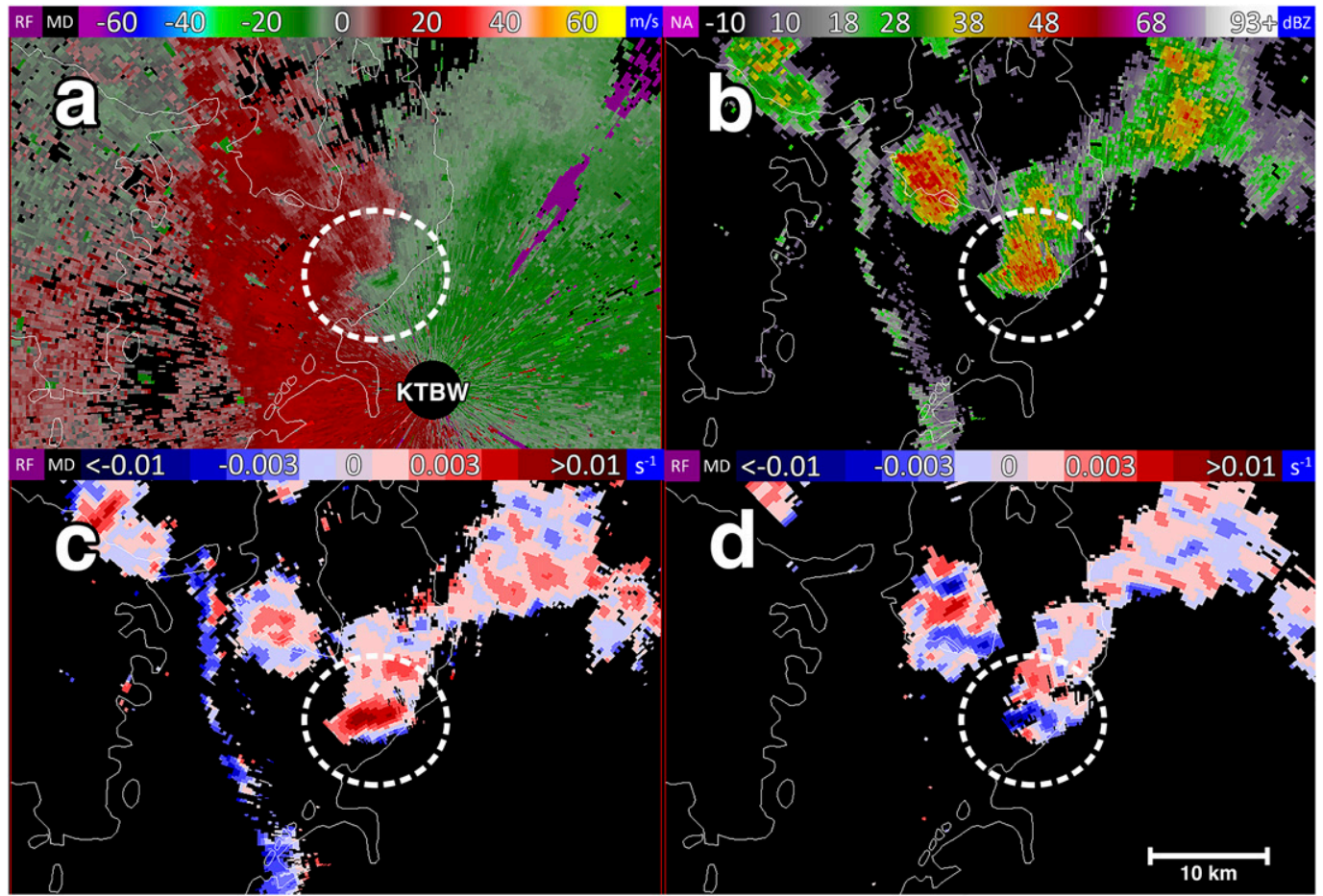

FIG. 7. Single-radar, $0.5^{\circ}$ (a) dealiased $V_{r}$, (b) QC $Z_{H}$, and (c) DivShear, and (d) $10.0^{\circ}$ DivShear showing the low-level divergence and midlevel convergence associated with a downburst (denoted by the dashed circle) sampled by the Tampa Bay, FL (KTBW), radar at 2219 UTC 24 Aug 2011. The DivShear in (c) and (d) are thresholded by $>10 \mathrm{dBZ} 0.5^{\circ} \mathrm{QC} Z_{H}$.

In general, a nonuniform weighting scheme will reduce the magnitudes of the derivatives, except when the kernel is centered on the underlying radar signature (e.g., a mesocyclone), where it will receive near-full weighting. Practically, nonuniform weighting results in increased isolation of features within the input field, which in turn increases gradients within the derivative fields. Internal testing (not shown) of kernel weighting revealed the output derivative fields more quickly yield background-level values as input field features are increasingly offset from the kernel center. Specific radar applications using the LLSD technique should explore the use of weighting schemes as part of their implementation.

\section{c. Validation}

Mathematically, when applied to a $V_{r}$ field, the slope of the LLSD best-fit plane across azimuth $\theta$ [Eq. (12a)] is represented as a simple rise over run $\delta V_{r} / \delta \theta$, which corresponds to one term of $\zeta$, assuming local symmetry. Thus, AzShear can be considered an approximation for one-half of the total $\zeta$. To evaluate this relationship and quantify the effects of kernel size on AzShear, calculations of AzShear were compared to theoretical half- $\zeta$, defined as $\Delta V_{r} / 2 r$ for a variety of simulated circulations. The $V_{r}$ signatures of simulated vortices of varying size, intensity, and range from a hypothetical WSR-88D superresolution radar $\left(0.5^{\circ}\right.$ azimuthal resolution and $1.02^{\circ}$ effective beamwidth) were generated using a Rankine combined vortex model (Wood and Brown 1997). Circulations were varied in radius $(1000-8000 \mathrm{~m}$ in increments of $250 \mathrm{~m})$, distance to radar $(10-200 \mathrm{~km}$ in increments of $5 \mathrm{~km}$ ), and circulation strength (delta- $V=$ $V_{\max }-V_{\min }$, from 10 to $50 \mathrm{~m} \mathrm{~s}^{-1}$ in increments of $5 \mathrm{~m} \mathrm{~s}^{-1}$ ). A total of 10179 vortices were simulated. Following the approach of SE04 and Miller et al. (2013), $2 \mathrm{~m} \mathrm{~s}^{-1}$ uniform noise was superimposed on the $V_{r}$ field of each to better simulate the imperfections present in real radar fields.

Each simulated vortex was passed through the LLSD algorithm to calculate AzShear using four different kernels, each with a constant radial depth of $750 \mathrm{~m}$ (since a radar's radial resolution should not change) but with different azimuthal widths: 1500, 2500, 5000, and $8000 \mathrm{~m}$. Maximum AzShear calculations for all circulations with theoretical half- $\zeta$ of $0.02 \mathrm{~s}^{-1}$, a common value for strongly rotating storms (Burgess et al. 1975), are summarized in Fig. 4. In general for the circulations within $90 \mathrm{~km}$ from the radar, a kernel $2500 \mathrm{~m}$ wide by $750 \mathrm{~m}$ deep produced the most accurate AzShear calculation, with the average maximum AzShear values varying from the theoretical shear by $5 \%$ or less (Fig. 5a). Beyond $90 \mathrm{~km}$, this kernel underestimated the values. A smaller kernel (1500-m azimuthal width) 


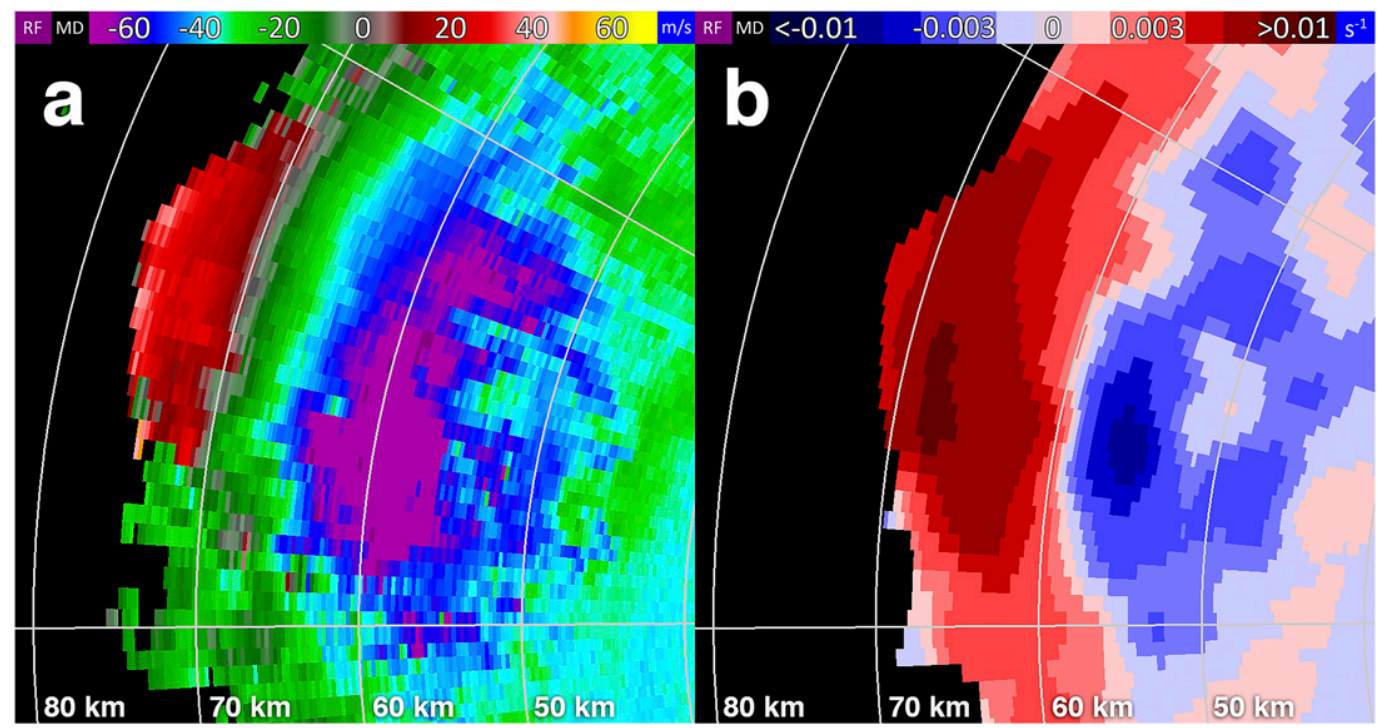

FIG. 8. Single-radar (a) $12.5^{\circ}$ dealiased $V_{r}$ and (b) corresponding DivShear showing a storm-top divergence signature associated with convection from the Oklahoma City, OK (KTLX), radar at 2313 UTC 31 May 2013. The divergence located at the top of the updraft is indicated by strongly positive DivShear and is flanked by a region of negative DivShear in (b). A kernel with an azimuthal width of $750 \mathrm{~m}$ and a radial depth of $8750 \mathrm{~m}$ was used.

tended to overestimate the rotation intensity of the circulations within $80 \mathrm{~km}$ of the radar by $10 \%-15 \%$ while larger kernels resulted in underestimations of $30 \%$ (5000-m azimuthal width) to $60 \%$ (8000-m azimuthal width) over the same radar distances. These results are caused by small kernels failing to include information from $V_{r}$ measurements outside of the vortex core and large kernels effectively smoothing the rotation across a larger area. Large kernels are also more sensitive to the physical size of the circulation and produce a wider range of AzShear calculations within $100 \mathrm{~km}$ of the radar, as illustrated by the large-area violin plots in
Figs. $4 \mathrm{c}$ and $4 \mathrm{~d}$ and large median-centered interquartile ranges (IQR; Fig. 5b).

Across a diverse set of simulated vortices, the kernel with an azimuthal width of $2500 \mathrm{~m}$ and radial depth of $750 \mathrm{~m}$ tended to best match theoretical $\zeta$, particularly for those with characteristics common to strong mesocyclones. This kernel size is most appropriate for real-time AzShear calculations given the authors' experience implementing it for severe weather analysis. However, analyses of phenomena of varying scales may find other kernel sizes more appropriate, such as a storm-top divergence signature. In general, larger kernels effectively

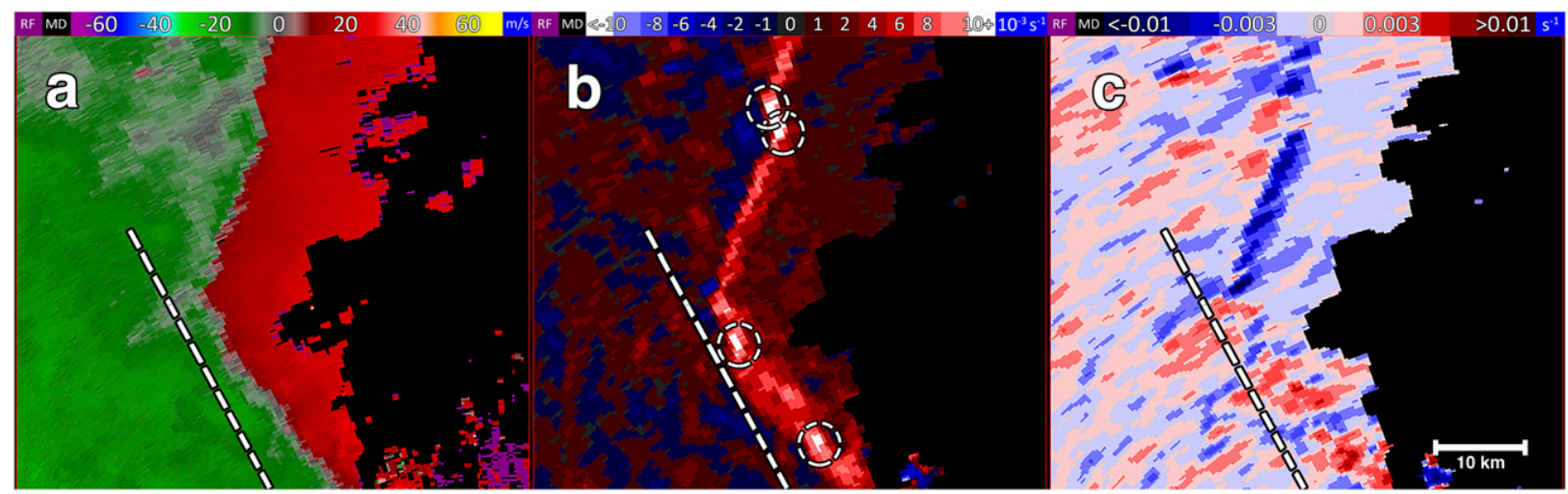

FIG. 9. Single radar, $0.5^{\circ}$ (a) dealiased $V_{r}$ and corresponding (b) AzShear and (c) DivShear associated with a QLCS from the Shreveport, LA (KSHV), radar at 0454 UTC14 Apr 2018. Strong convergence (blue) occurs along the line in (c) but is not highlighted as such when it is aligned approximately parallel to the KSHV radar beam (approximated by the dashed line). Note several locations of embedded rotation in the AzShear field in (b), denoted by the dashed circles. 


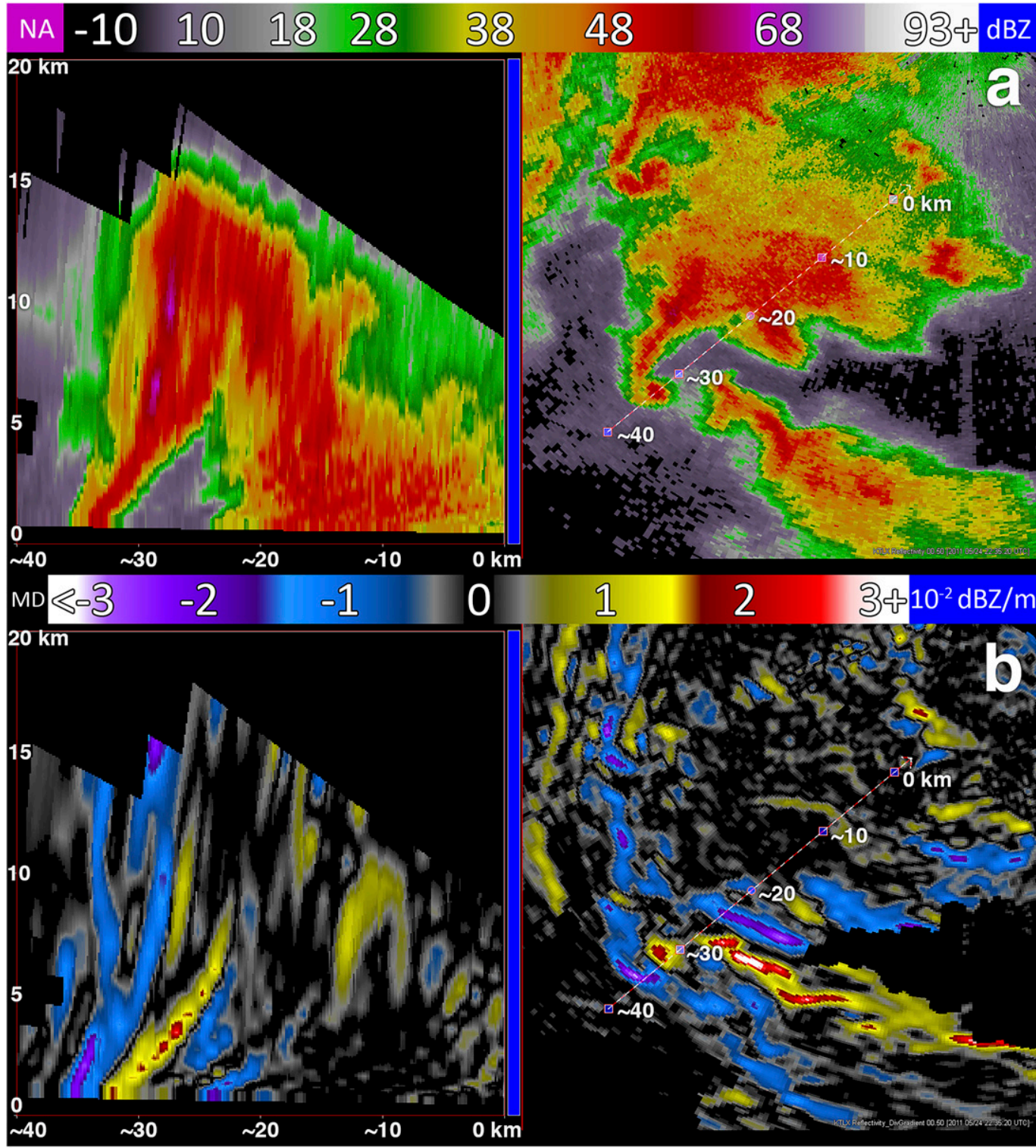

FIG. 10. Single-radar (a) $Z_{H}$ and (b) corresponding LLSD azimuthal $Z_{H}$ gradient for a tornadic supercell from KTLX at 2235 UTC 24 May 2011. (left) A vertical cross-section taken along the line depicted in the (right) corresponding $0.5^{\circ} \mathrm{PPI}$. This multidimensional application of $Z_{H}$ gradients and the additional context provided by the sign of the gradient can be used to interrogate storm structure.

filter the data more, retaining only the strongest or large-scale gradients. Large-scale derivatives are not removed by smaller kernels, but they may be masked by small-scale gradients that are not resolvable by a larger kernel.

The results shown in Figs. 4-5 are averaged over a representative subset of simulations. A comprehensive analysis of AzShear accuracy relative to small changes in vortex size, strength, and range from radar is beyond the scope of this paper and will be left for future study.

\section{Applications}

\section{a. Velocity}

AzShear and DivShear are the LLSD gradients of $V_{r}$ fields (Fig. 1) and represent an approximation for half- $\zeta$ and half-divergence, respectively. Positive (negative) AzShear values represent cyclonic (anticyclonic) rotation, while positive (negative) DivShear represents divergence (convergence).

Using a different method of calculating AzShear, Burgess et al. (1975) first developed ranges of typical 
AzShear values that still largely hold true: AzShear often surpasses $\pm 0.01 \mathrm{~s}^{-1}$ in strong circulations, and the most intense circulations can exceed $\pm 0.05 \mathrm{~s}^{-1}$. Since values tend to be maximized in tornadic supercells (Burgess et al. 1975), AzShear is especially useful for rotation identification and tracking (Manross et al. 2008; Karstens et al. 2016), which, in turn, is valuable for TDS identification (Ryzhkov et al. 2005; Snyder and Ryzhkov 2015) and model verification (Skinner et al. 2016).

The LLSD technique has the ability to detect rotation at different spatial scales, with or without changing the kernel size. Data surrounding $V_{r}$ extrema of any kind will be associated with nonzero AzShear since the extrema are, by definition, greater than the surrounding winds, thus producing a gradient between the two. For example, Fig. 6 shows enhanced AzShear near the strongest winds within the eyewall of a tropical cyclone (Fig. 6b), while also highlighting rotating small-scale features such as tornadic circulations embedded in outer rainbands (Figs. 6d,e), which are often difficult to detect using traditional radarbased algorithms (Spratt et al. 1997).

DivShear was originally used in the development of an experimental damaging downburst detection algorithm (Smith et al. 2004) due to its ability to identify downburst signatures of converging flow aloft and diverging flow near the surface (Fig. 7). It can be also used to identify and investigate supercell structure (Fig. 1; Heinselman et al. 2008) and mesoscale features such as sea breezes and outflow boundaries. Larger-scale divergent features, such as storm-top divergence (Fig. 8), can be examined using LLSD kernels with increased radial depth.

Unlike rotation signatures, which are spatially fairly compact and nearly circular, elongated, quasi-linear features like convergence boundaries are a function of radar viewing angle. Along-azimuth gradients are minimized when linear features are oriented parallel to the radar beam, meaning that DivShear values are inherently a function of the feature's orientation to the radar. For example, the quasi-linear convective system (QLCS) depicted in Fig. 9, which produced severe winds and occasional tornadoes, is a line of convergent $V_{r}$ (Fig. 9a) and an elongated region of AzShear (Fig. 9b). However, the QLCS curves such that its southern half is aligned approximately parallel to the beam, resulting in very little along-azimuth gradient, and locally weak DivShear (Fig. 9c). Given these considerations, some form of the total derivative, or another combination of both shear components, may be useful when examining features with high aspect ratio (e.g., noncircular features such as gust fronts). Interrogating single-radar data from another radar with a different viewing angle of the same feature, or merging data from more than one radar, may also help alleviate this issue.

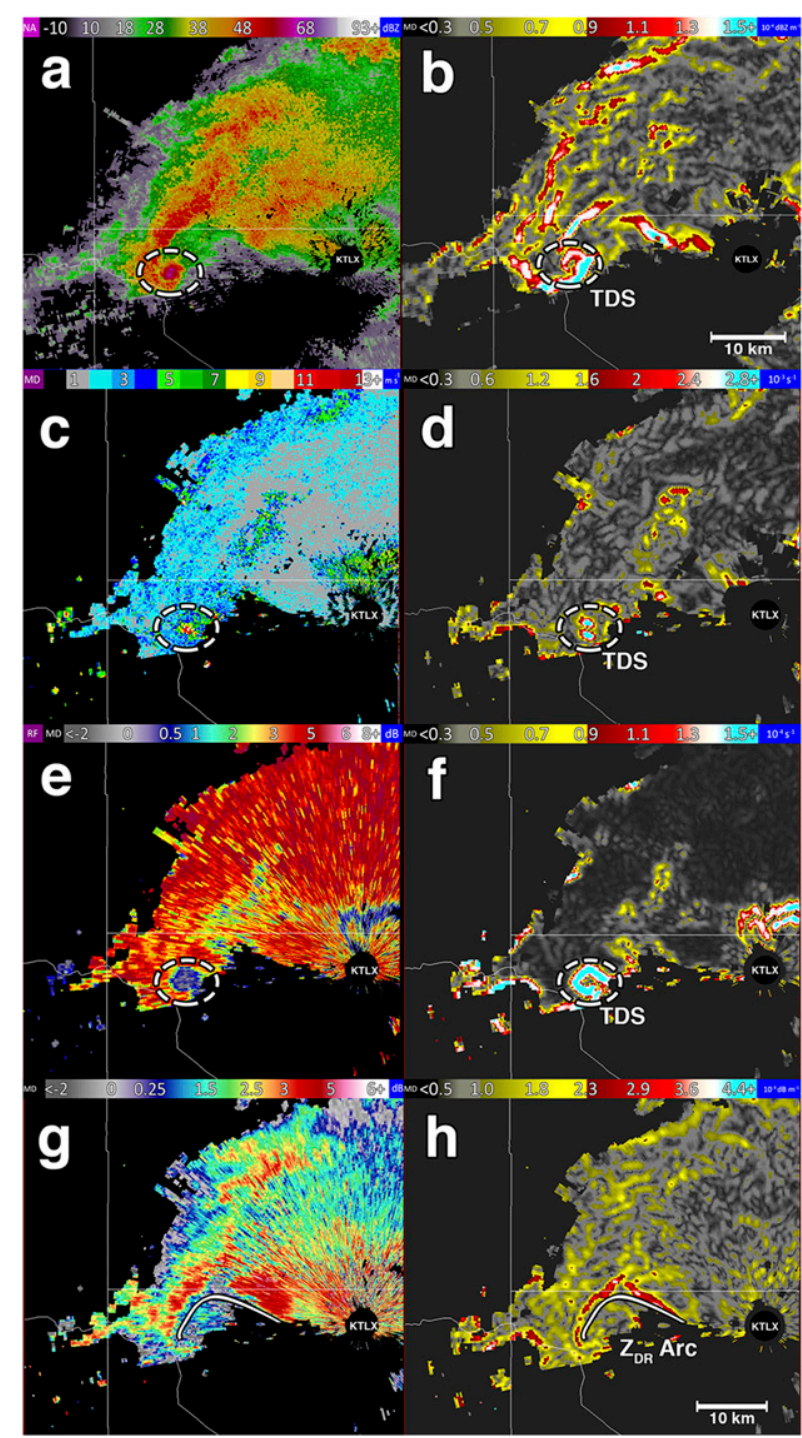

FIG. 11. Single-radar, $0.5^{\circ}$ (a) $\mathrm{QC} Z_{H}$, (c) spectrum width, (e) $\rho_{\mathrm{hv}}$, and (g) $Z_{\mathrm{DR}}$, and (b),(d),(f),(h) corresponding LLSD gradients of each for a tornadic supercell, from KTLX at 2012 UTC 20 May 2013. Polarimetric fields were passed through a preprocessor and thresholded by $>20 \mathrm{~dB} Z$ QC $Z_{H}$. The gradients in (d) and (f) are largest in the vicinity of the tornadic debris signature (denoted by the dashed ellipse) associated with a significant tornado at the time of this scan. The gradient in (h) (denoted by the arched line) highlights a $Z_{\mathrm{DR}}$ arc.

Operationally, AzShear is produced within the MRMS system at the National Centers for Environmental Prediction. It is first calculated as a single-radar, layer-maximum product and then is merged onto the MRMS continental United States (CONUS) grid. For example, layer-maximum AzShear is the maximum AzShear within a vertical layer. For the operational MRMS system, two vertical layers are used: low-level [0-2 km above ground level (AGL)] and midlevel 


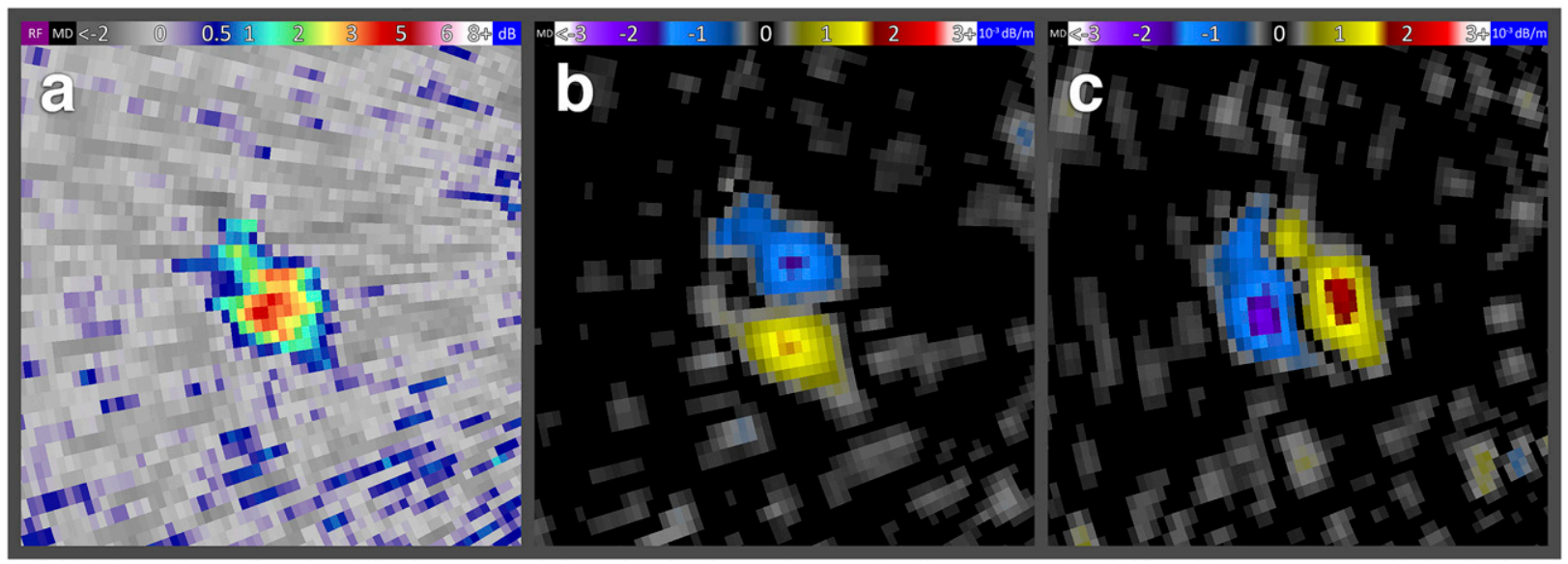

FIG. 12. Single-radar (a) $12.5^{\circ} Z_{\mathrm{DR}}$ and corresponding (b) azimuthal and (c) radial LLSD gradients of a $Z_{\mathrm{DR}}$ column within a storm from the Sioux Falls, SD (KFSD), radar at 0352 UTC 18 Jun 2014. Both LLSD gradient components surround the core of a $Z_{\mathrm{DR}}$ maximum. Tracking these LLSD gradient peaks over several elevation scans in the same volume can be used to aid in the identification of a $Z_{\mathrm{DR}}$ column.

(3-6 km AGL; Smith et al. 2016). Layered AzShear is accumulated over time to produce low- and midlevel rotation tracks (as in Fig. 2), the standard MRMS products used to visualize the paths and intensity trends of mesocyclones (Miller et al. 2013).

\section{b. Reflectivity}

For scalar fields, the individual azimuthal and radial LLSD gradients of $Z_{H}$ and other non- $V_{r}$ radar fields often provide less meaningful information than $V_{r}$ gradients but may help reveal features when examined in unison. One method to calculate the total, absolute magnitude of an LLSD gradient is found by calculating the vector norm of the local gradient:

$$
\mathbf{u}=\sqrt{u_{\theta}^{2}+u_{r}^{2}} .
$$

Total $Z_{H}$ gradients can help identify substorm-scale features such as supercell bounded weak echo regions (BWER) and the vertical structure of precipitation cores (Fig. 10), providing three-dimensional details of $Z_{H}$ distributions that may help diagnose characteristics of precipitation type, heavy rainfall, hail, or other nonmeteorological scatterers.

The total, horizontal gradient [Eq. (13)] of $Z_{H}\left(\mathrm{~dB} Z \mathrm{~m}^{-1}\right)$ effectively produces a $Z_{H}$ "stencil," outlining individual storms or $Z_{H}$ clusters (Figs. 11a,b) by highlighting sharp changes in $Z_{H}$, which themselves can be caused by a wide range of meteorological phenomena. For example, a supercell's shape and the debris ball $Z_{H}$ signature (a $Z_{H}$ maximum resulting from lofted debris; Bunkers and Baxter 2011) are seen in the $Z_{H}$ gradients in Fig. 11b. LLSD $Z_{H}$ gradient calculations are sensitive to ground clutter contamination, however, and any nonmeteorological $Z_{H}$ not removed by qualitycontrol algorithms may interfere with the meteorological signal (Lakshmanan et al. 2007a), as evidenced by isolated, spuriously high LLSD gradient values in Fig. 11 and other nonvelocity gradient figures.

One potential application of LLSD-derived $Z_{H}$ gradient information could include its implementation within hydrometeor classification or discrimination algorithms. For instance, large hail sizes (diameters exceeding $25 \mathrm{~mm}$ ) have been observed in low $Z_{H}$ regions (below 40 dBZ; e.g., Snyder et al. 2014, 2017), while operational algorithms typically use minimum $Z_{H}$ values at or above $40 \mathrm{dBZ}$ for hail classification and sizing (Witt et al. 1998; Park et al. 2009; Ortega et al. 2016). Use of $Z_{H}$ gradients instead of or along with simple $Z_{H}$ thresholds may help improve the skill of these algorithms. The first potential avenue for improvement would be to incorporate the gradient fields into the validation of the algorithms. For instance, Ortega et al. (2016) used only peak reflectivity values at the report point, or within a surrounding neighborhood, to determine the best match for validation. Adding the gradient field might help with more precise matching of reports to algorithm outputs, or at least further stratify results to better explain algorithm skill. The second way would be to directly incorporate gradient fields into the algorithms should they show discrimination power.

\section{c. Spectrum width and polarimetric moments}

Gradients of $\rho_{\mathrm{hv}}\left(\mathrm{m}^{-1}\right)$ and spectrum width $\left(\mathrm{s}^{-1}\right)$ can help indicate the presence of a TDS by highlighting tight gradients surrounding a region of extreme values (Figs. 11c-f; Zrnić and Doviak 1975; Ryzhkov et al. 2005). In some cases, gradients related to important storm properties can visually appear obvious, and thus be useful 


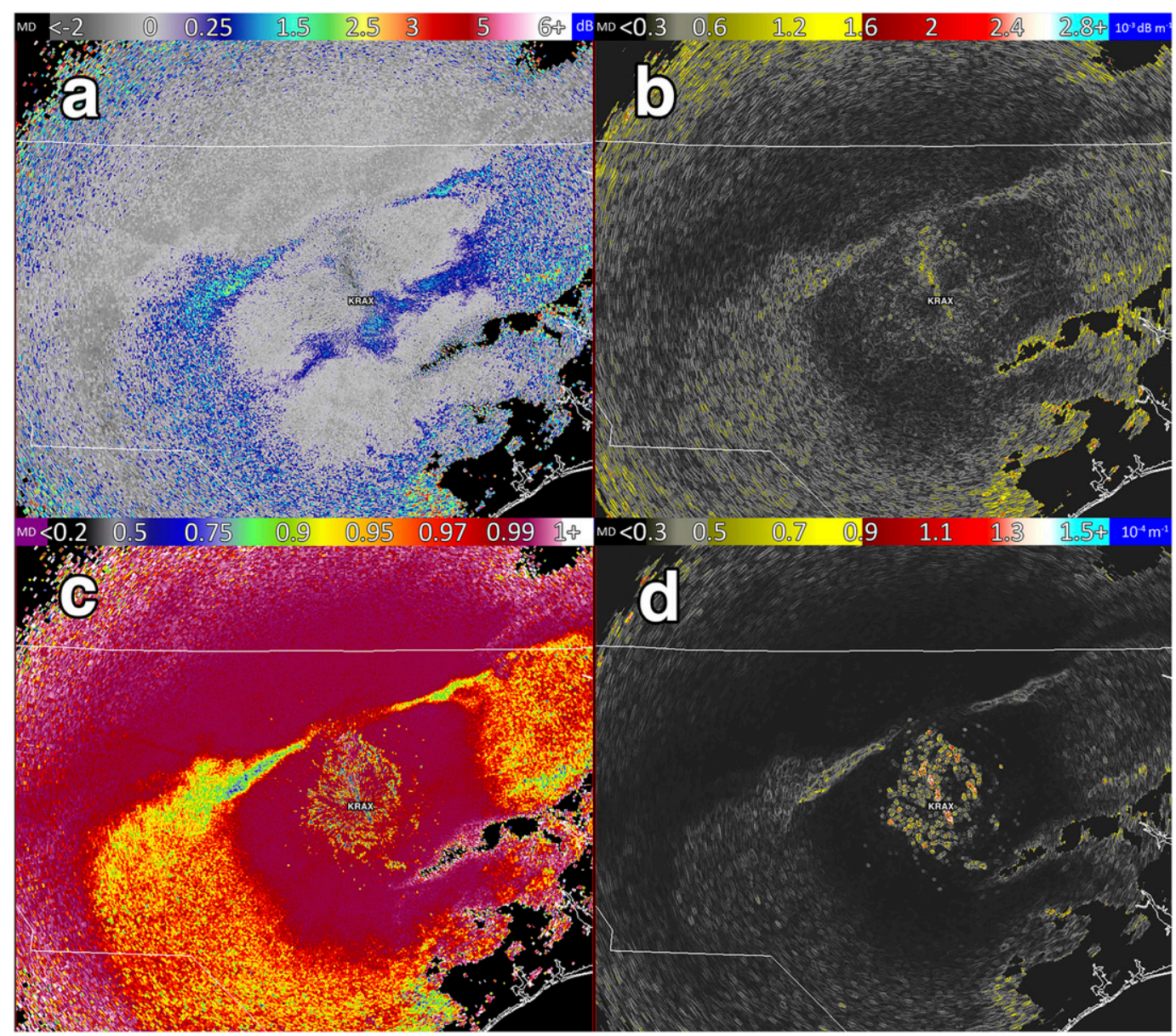

FIG. 13. Single-radar, $0.5^{\circ}$, preprocessed (a) $Z_{\mathrm{DR}}$ and (c) $\rho_{\mathrm{hv}}$ and (b),(d) corresponding total (i.e., the norm of both components) LLSD gradients of each observed during a winter storm with multiple precipitation types across North Carolina, from the Raleigh/Durham, NC (KRAX), radar at 0310 UTC 7 Jan 2017. Poor $\rho_{\text {hv }}$ data quality causes the noisy data near the radar in (c) and (d). In both instances, changes in the relative homogeneity of the gradient fields help distinguish between precipitation types.

during operational applications. At this time, there is no obvious added benefit to using LLSD gradient fields directly in an operational setting, particularly in short-fuse situations, when compared to simply using the human eye. However, these fields reveal patterns in gradients that may be useful in future application development. The examples of polarimetric variable gradients shown here were generated from data processed through the WSR88D preprocessor (Istok et al. 2009), which smooths the polarimetric variables along the radial and applies corrections to $Z_{\mathrm{DR}}$ for attenuation and system calibrations.

In well-organized supercells, $Z_{\mathrm{DR}}$ gradients $\left(\mathrm{dB} \mathrm{m}^{-1}\right)$ can highlight $Z_{\mathrm{DR}}$ arcs (Figs. 11g,h; Kumjian and Ryzhkov 2008) and $Z_{\mathrm{DR}}$ columns (Fig. 12; Kumjian and Ryzhkov 2008), which are related to the magnitude of storm-relative helicity and may diagnose relative updraft strength and hail production, respectively (Kumjian and Ryzhkov 2009). Identifying and monitoring the trends of these characteristics can help in tracking the location and intensity of individual storms and their hazards.

Gradients of $Z_{\mathrm{DR}}$ and $\rho_{\mathrm{hv}}$ also show the melting (Ryzhkov et al. 1998) and/or refreezing layers (Kumjian et al. 2013) within both severe convective storms and synopticscale winter precipitation (Ryzhkov and Zrnić 1998) and may help diagnose frozen and mixed-phase cloud physical processes. Values of these products are generally fairly constant across areas of pure snow, while mixed precipitation fields are more heterogeneous due to variations in hydrometeor size and shape. Total LLSD gradients of $Z_{\mathrm{DR}}$ and $\rho_{\mathrm{hv}}$ detect mixed-phase layers by their heightened spatial variability: many finescale gradients occur within mixed-phase regions, while relatively homogenous precipitation regions contain very few (Fig. 13). Because of the relatively noisy nature of polarimetric data, larger LLSD kernels effectively filter out these signals over a large area. 


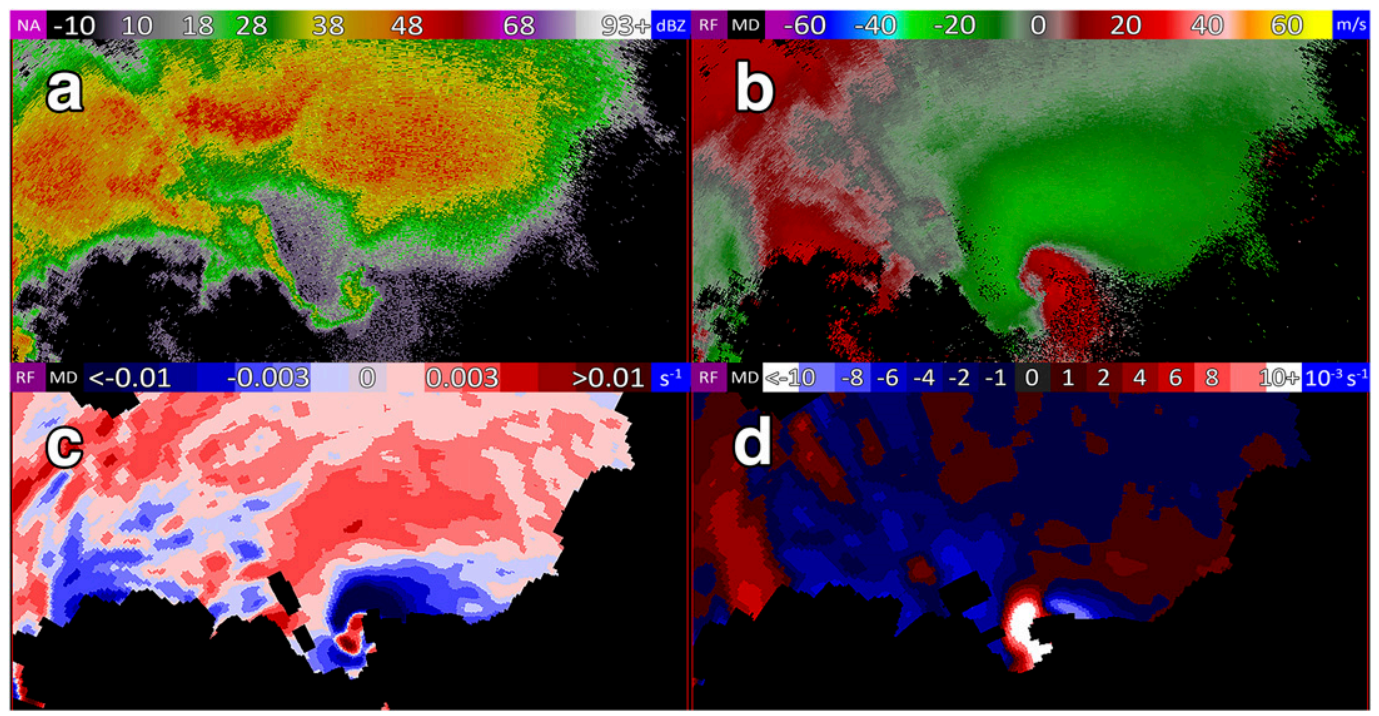

FIG. 14. Single-radar, $0.5^{\circ}$ (a) $Z_{H}$, (b) dealiased $V_{r}$, (c) DivShear, and (d) AzShear from a NOXP sweep of a supercell in western Nebraska at 1659 UTC 13 Jun 2017. Missing DivShear and AzShear in (c) and (d) are caused by missing or range folded data being present in within the LLSD kernel.

Although more analysis is needed to quantify the relationships between non- $V_{r}$ LLSD gradients and the physical processes described above, it is possible that these gradient calculations, particularly when examined in conjunction with other meteorological data, may aid in ongoing research efforts such as visualizing internal storm processes and isolating regions of particular hydrometeor regimes. Such LLSD gradients may prove especially useful for automated feature detection methods, for example, as input predictors in machine learning applications such as random forest (Breiman 2001) or deep learning (Schmidhuber 2015) classification or identification techniques. Implementation and evaluation of these potential applications will be left to future work. LLSD $V_{r}$ gradients have already been implemented in the development of new mesocyclone and tornado detection algorithms for the WSR-88D network (Ortega et al. 2018).

\section{d. Radar types}

The LLSD algorithms used at NSSL have been optimized to process data from the Weather Surveillance Radar-1988 Doppler (WSR-88D) S-band radars. These radars have changed resolutions over time from legacy $\left(1.38^{\circ}\right.$ effective beamwidth; Brown et al. 2002, 2005) to super-resolution $\left(1.02^{\circ}\right.$ effective beamwidth; Torres and Curtis 2007) scanning strategies. The algorithm has been successfully tested on both resolutions, though the examples provided in this section apply specifically to super-resolution.

The LLSD equations do not change for data from other, non-WSR-88D systems, such as mobile, C-band, and X-band radars, regardless of spatial or temporal resolution. The algorithm described here has undergone testing on data from a variety of platforms, including the Rapid-scan X-band Polarimetric mobile radar (RaXPol; Pazmany et al. 2013; Wienhoff et al.2017) and the NOAA X-band dual-polarized (NOXP; Burgess et al. 2010) mobile radar (Fig. 14), and calculated gradients satisfactorily for each. In addition, LLSD kernel sizes can be manually modified for different radar characteristics, if desired.

\section{e. Data quality}

Although the LLSD approach is designed to minimize the effects of poor data quality, it is still subject to the quality of its input field. Operational AzShear blocks out measurements associated with range folding or missing data but will otherwise complete LLSD calculations on the input $V_{r}$ field. These calculations may be negatively affected by a number of data quality issues, including sidelobe contamination (Piltz and Burgess 2009), dealiasing failures (Figs. 15a,b; Witt et al. 2009), wind farms or other ground clutter (Figs. 15c,d; Isom et al. 2007), and others. For example, in Fig. 15a, artificial AzShear spikes exist along the edges of dealiasing failures, where folds between Nyquist velocity intervals occur. However, since all $V_{r}$ values elsewhere within the folded region are uniformly affected by the failure, $V_{r}$ gradients are correct.

\section{Summary and future work}

An updated derivation and new applications of the improved LLSD approach to calculating gradients in radar data fields have been presented, along with a comprehensive 

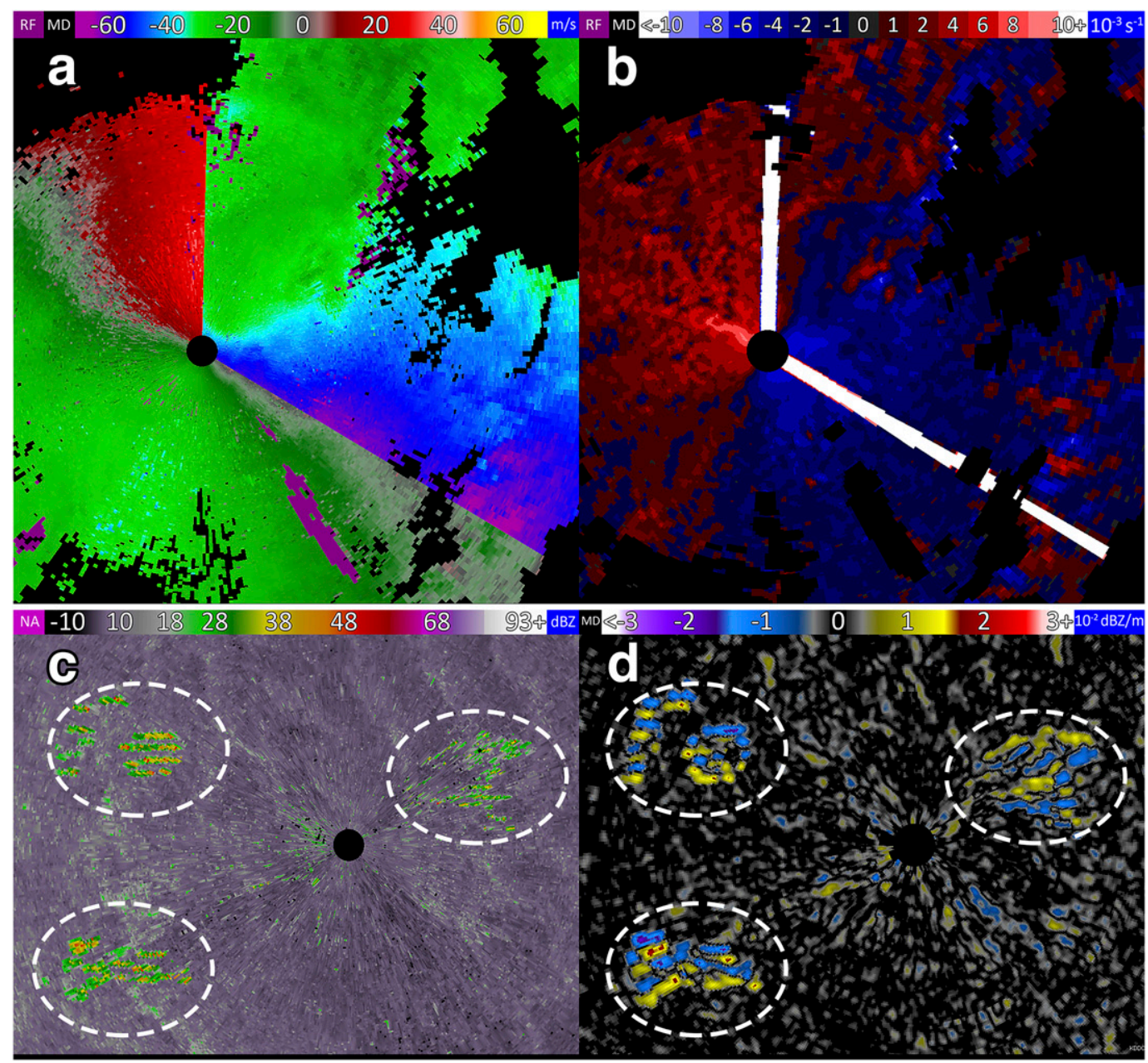

FIG. 15. Examples of poor data quality affecting LLSD gradient calculations. (top) Single-radar (a) $3.1^{\circ}$ dealiased $V_{r}$ and (b) corresponding AzShear from the Kansas City/Pleasant Hill, MO (KEAX), radar at 0221 UTC 20 May 2013. Nonphysical, extreme AzShear occurs along straight lines surrounding a dealiasing failure. (bottom) Singleradar (c) $0.9^{\circ} Z_{H}$ and (d) corresponding LLSD azimuthal $Z_{H}$ gradient from the Dodge City, KS (KDDC), radar at 2147 UTC 24 May 2016. The $Z_{H}$ returns are caused by wind farms (denoted by the dashed circles).

discussion of considerations for technique application. The methodology described here can be applied to nearly any radar data field for use in interrogating a wide variety of meteorological phenomena, whether in a manual, userdriven warning-decision sense or perhaps an automated, computer-driven, object-based approach. While up to this point most applications have focused on detecting rotation (AzShear) or aviation hazards (DivShear), LLSD output also may be used as a new approach to depicting storm structure, severe weather signatures, and microphysical processes. However, the work shown here is intended to provide an overview of potential applications, and these analyses represent the minimum that should be done when completing a quantitative analysis of a LLSD product. Use of any LLSD output should be thoroughly tested and evaluated when used in any application.
As with all radar-derived analysis techniques, LLSD products are subject to standard radar sampling limitations. Future work should include thorough documentation of these limitations for specific LLSD applications and an investigation into overcoming them. This may include revisiting previous methods of alleviating range effects (Newman et al. 2013), additional exploration using different kernel sizes for various, feature-specific identifications, and a more expansive investigation of potential kernel weighting techniques.

All LLSD examples in this paper were produced using single-Doppler radar data, but similar multiradar products generated within the MRMS framework should prove useful as well. Thus, the effects of multiradar mergers of LLSD fields, including AzShear, should also be investigated. In addition, further scrutiny of many of 
the signatures presented here is necessary, and future work should be performed to test their prevalence.

Acknowledgments. The authors thank Don Burgess for supplying NOXP radar data and assisting in the development of this manuscript. We also thank Vincent Wood and the anonymous reviewers for providing helpful feedback. Funding was provided by NOAA/ Office of Oceanic and Atmospheric Research under NOAA-University of Oklahoma Cooperative Agreement NA11OAR4320072, U.S. Department of Commerce.
Additional, partial funding for this work was provided by a Memorandum of Understanding between NSSL and the NEXRAD Program, managed by the Radar Operations Center (ROC).

\section{APPENDIX A}

\section{Complete LLSD Azimuthal Gradient Equation}

Complete form of the LLSD azimuthal shear (AzShear) equation:

$$
\begin{aligned}
u_{\theta}= & \left(\sum_{k=0}^{m \times n} \Delta r_{k}^{2} \sum_{k=0}^{m \times n} \Delta \theta_{k}^{2} \sum_{k=0}^{m \times n} w_{k}-\sum_{k=0}^{m \times n} \Delta \theta_{k}^{2} \sum_{k=0}^{m \times n} \Delta r_{k} \sum_{k=0}^{m \times n} \Delta r_{k}\right. \\
& +2 \sum_{k=0}^{m \times n} \Delta r_{k} \Delta \theta_{k} \sum_{k=0}^{m \times n} \Delta r_{k} \sum_{k=0}^{m \times n} \Delta \theta_{k}-\sum_{k=0}^{m \times n} \Delta r_{k}^{2} \sum_{k=0}^{m \times n} \Delta \theta_{k} \sum_{k=0}^{m \times n} \Delta \theta_{k} \\
& \left.-\sum_{k=0}^{m \times n} w_{k} \sum_{k=0}^{m \times n} \Delta r_{k} \Delta \theta_{k} \sum_{k=0}^{m \times n} \Delta r_{k} \Delta \theta_{k}\right)^{-1}\left[\sum _ { k = 0 } ^ { m \times n } \Delta r _ { k } u _ { k } \left(\sum_{k=0}^{m \times n} \Delta r_{k}^{2} \sum_{k=0}^{m \times n} w_{k}\right.\right. \\
& \left.-\sum_{k=0}^{m \times n} \Delta \theta_{k} \sum_{k=0}^{m \times n} \Delta \theta_{k}\right)+\sum_{k=0}^{m \times n} \Delta \theta_{k} u_{k}\left(\sum_{k=0}^{m \times n} \Delta r_{k} \sum_{k=0}^{m \times n} \Delta \theta_{k}-\sum_{k=0}^{m \times n} \Delta r_{k} \Delta \theta_{k} \sum_{k=0}^{m \times n} w_{k}\right) \\
& \left.+\sum_{k=0}^{m \times n} u_{k}\left(\sum_{k=0}^{m \times n} \Delta r_{k} \Delta \theta_{k} \sum_{k=0}^{m \times n} \theta_{k}^{2}-\sum_{k=0}^{m \times n} \Delta r_{k} \sum_{k=0}^{m \times n} \Delta \theta_{k}^{2}\right)\right],
\end{aligned}
$$

where $k$ is the index for each kernel grid point, $m$ and $n$ are the number of grid points in the LLSD kernel in $x(m)$ and $y(n), \Delta r_{k}$ is the radial distance from the center of the LLSD kernel, $\Delta \theta_{k}$ is the azimuthal distance from the center of the LLSD kernel, $u_{k}$ is the radar variable measurement at kernel grid point $k$, and $w_{k}$ is the local weight at kernel grid point $k$.

\section{APPENDIX B}

\section{Complete LLSD Radial Gradient Equation}

Complete form of the LLSD radial shear (DivShear) equation:

$$
\begin{aligned}
u_{r}= & \left(\sum_{k=0}^{m \times n} \Delta r_{k}^{2} \sum_{k=0}^{m \times n} \Delta \theta_{k}^{2} \sum_{k=0}^{m \times n} w_{k}-\sum_{k=0}^{m \times n} \Delta \theta_{k}^{2} \sum_{k=0}^{m \times n} \Delta r_{k} \sum_{k=0}^{m \times n} \Delta r_{k}+2 \sum_{k=0}^{m \times n} \Delta r_{k} \Delta \theta_{k} \sum_{k=0}^{m \times n} \Delta r_{k} \sum_{k=0}^{m \times n} \Delta \theta_{k}\right. \\
& -\sum_{k=0}^{m \times n} \Delta r_{k}^{2} \sum_{k=0}^{m \times n} \Delta \theta_{k} \sum_{k=0}^{m \times n} \Delta \theta_{k} \\
& \left.-\sum_{k=0}^{m \times n} w_{k} \sum_{k=0}^{m \times n} \Delta r_{k} \Delta \theta_{k} \sum_{k=0}^{m \times n} \Delta r_{k} \Delta \theta_{k}\right)^{-1}\left[\sum _ { k = 0 } ^ { m \times n } \Delta r _ { k } u _ { k } \left(\sum_{k=0}^{m \times n} \Delta r_{k} \sum_{k=0}^{m \times n} \Delta \theta_{k}\right.\right. \\
& \left.-\sum_{k=0}^{m \times n} w_{k} \sum_{k=0}^{m \times n} \Delta r_{k} \Delta \theta_{k}\right)+\sum_{k=0}^{m \times n} \Delta \theta_{k} u_{k}\left(\sum_{k=0}^{m \times n} \Delta r_{k}^{2} \sum_{k=0}^{m \times n} w_{k}-\sum_{k=0}^{m \times n} \Delta r_{k} \sum_{k=0}^{m \times n} \Delta r_{k}\right) \\
& \left.+\sum_{k=0}^{m \times n} u_{k}\left(\sum_{k=0}^{m \times n} \Delta r_{k} \Delta \theta_{k} \sum_{k=0}^{m \times n} \Delta r_{k}-\sum_{k=0}^{m \times n} \Delta \theta_{k} \sum_{k=0}^{m \times n} \Delta r_{k}^{2}\right)\right],
\end{aligned}
$$

where $k$ is the index for each kernel grid point, $m$ and $n$ are the number of grid points in the LLSD kernel in $x(m)$ and $y(n), \Delta r_{k}$ is the radial distance from the center of the LLSD kernel, $\Delta \theta_{k}$ is the azimuthal distance from 
the center of the LLSD kernel, $u_{k}$ is the radar variable measurement at kernel grid point $k$, and $w_{k}$ is the local weight at kernel grid point $k$.

\section{REFERENCES}

Alexander, C. R., and J. Wurman, 2008: Updated mobile radar climatology of supercell tornado structures and dynamics. 24th Conf. on Severe Local Storms, Savannah, GA, Amer. Meteor. Soc., 19.4, http://ams.confex.com/ams/pdfpapers/141821.pdf.

Breiman, L., 2001: Random forests. Mach. Learn., 45, 5-32, https:// doi.org/10.1023/A:1010933404324.

Brown, R. A., L. R. Lemon, and D. W. Burgess, 1978: Tornado detection by pulsed Doppler radar. Mon. Wea. Rev., 106, 29-38, https://doi.org/10.1175/1520-0493(1978)106<0029: TDBPDR $>2.0 . \mathrm{CO} ; 2$.

- , V. T. Wood, and D. Sirmans, 2002: Improved tornado detection using simulated and actual WSR-88D data with enhanced resolution. J. Atmos. Oceanic Technol., 19, 1759-1771, https://doi.org/10.1175/1520-0426(2002)019<1759: ITDUSA $>2.0 . \mathrm{CO} ; 2$

, B. A. Flickinger, E. Forren, D. M. Schultz, D. Sirmans, P. L. Spencer, V. T. Wood, and C. L. Ziegler, 2005: Improved detection of severe storms using experimental fine-resolution WSR-88D measurements. Wea. Forecasting, 20, 3-14, https:// doi.org/10.1175/WAF832.1.

Bunkers, M. J., and M. A. Baxter, 2011: Radar tornadic debris signatures on 27 April 2011. Electron. J. Oper. Meteor., 12, 1-6.

Burgess, D. W., L. R. Lemon, and R. A. Brown, 1975: Tornado characteristics revealed by Doppler radar. Geophys. Res. Lett., 2, 183-184, https://doi.org/10.1029/GL002i005p00183.

— , R. J. Donaldson Jr., and P. R. Desrochers, 1993: Tornado detection and warning by radar. The Tornado: Its Structure, Dynamics, Prediction, and Hazards, Geophys. Monogr., Vol. 79, Amer. Geophys. Union, 203-221.

—, M. A. Magsig, J. Wurman, D. C. Dowell, and Y. Richardson, 2002: Radar observations of the 3 May 1999 Oklahoma City tornado. Wea. Forecasting, 17, 456-471, https://doi.org/ 10.1175/1520-0434(2002)017<0456:ROOTMO >2.0.CO;2.

, E. R. Mansell, C. M. Schwarz, and B. J. Allen, 2010: Tornado and tornadogenesis events seen by the NOXP X-band, dualpolarization radar during VORTEX2 2010.25th Conf. on Severe Local Storms, Denver, CO, Amer. Meteor. Soc., 5.2, http:// ams.confex.com/ams/25SLS/techprogram/paper_176164.htm.

—_ and Coauthors, 2014: 20 May 2013 Moore, Oklahoma, tornado: Damage survey and analysis. Wea. Forecasting, 29, 1229-1237, https://doi.org/10.1175/WAF-D-14-00039.1.

Carbone, R. G., J. W. Conway, N. A. Crook, and M. W. Moncrief, 1990: The generation and propagation of a nocturnal squall line, Part I: Observations and implications for mesoscale predictability. Mon. Wea. Rev., 118, 26-49, https://doi.org/ 10.1175/1520-0493(1990)118<0026:TGAPOA > 2.0.CO;2.

Cressman, G. P., 1959: An operational objective analysis system. Mon. Wea. Rev., 87, 367-374, https://doi.org/10.1175/15200493(1959)087<0367:AOOAS > 2.0.CO;2.

Davis, J. M., and M. D. Parker, 2014: Radar climatology of tornadic and nontornadic vortices in higher-shear, low-CAPE environments in the mid-Atlantic and southeastern United States. Wea. Forecasting, 29, 828-853, https://doi.org/10.1175/WAFD-13-00127.1.

Dawson, L. C., G. S. Romine, R. J. Trapp, and M. E. Baldwin, 2017: Verifying supercellular rotation in a convection-permitting ensemble forecasting system with radar-derived rotation track data. Wea. Forecasting, 32, 781-795, https://doi.org/10.1175/ WAF-D-16-0121.1.

Donaldson, R. J., Jr., 1970: Vortex signature recognition by a Doppler radar. J. Appl. Meteor., 9, 661-670, https://doi.org/ 10.1175/1520-0450(1970)009<0661:VSRBAD>2.0.CO;2.

_ 1978: Observations of the Union City tornadic storm by plan shear indicator. Mon. Wea. Rev., 106, 39-47, https://doi.org/ 10.1175/1520-0493(1978)106<0039:OOTUCT>2.0.CO;2.

Elmore, K. E., E. D. Albo, R. K. Goodrich, and D. J. Peters, 1994: NASA/NCAR airborne and ground-based wind shear studies. Final Rep., Contract NCC1-155, 343 pp.

Fujita, T. T., 1973: Proposed mechanism of tornado formation from rotating thunderstorms. Preprints, Eighth Conf. on Severe Local Storms, Denver, CO, Amer. Meteor. Soc., 191-196.

Heinselman, P. L., D. L. Priegnitz, K. L. Manross, T. M. Smith, and R. W. Adams, 2008: Rapid sampling of severe storms by the National Weather Radar Testbed Phased Array Radar. Wea. Forecasting, 23, 808-824, https://doi.org/10.1175/ 2008WAF2007071.1.

— D. S. LaDue, and H. Lazrus, 2012: Exploring impacts of rapid-scan radar data on NWS warning decisions. Wea. Forecasting, 27, 1031-1044, https://doi.org/10.1175/WAF-D11-00145.1.

Huang, T., G. Yang, and G. Tang, 1979: A fast two-dimensional median filtering algorithm. IEEE Trans. Acoust. Speech Signal Process., 27, 13-18, https://doi.org/10.1109/TASSP.1979.1163188.

Isom, B. M., and Coauthors, 2007: Characterization and mitigation of wind turbine clutter on the WSR-88D network. 33rd Conf. on Radar Meteorology, Cairns, Queensland, Australia, Amer. Meteor. Soc., 8B.8, https://ams.confex.com/ams/33Radar/ webprogram/Paper123300.html.

Istok, M. J., and Coauthors, 2009: WSR-88D dual polarization initial operating capabilities. 25th Conf. on Int. Interactive Information and Processing Systems (IIPS) for Meteorology, Oceanography, and Hydrology, Phoenix, AZ, Amer. Meteor. Soc., 15.5, http://ams.confex.com/ams/pdfpapers/ 148927.pdf.

Karstens, C. D., and Coauthors, 2016: Evaluation of near real-time preliminary tornado damage paths. J. Oper. Meteor., 4, 132-141, https://doi.org/10.15191/nwajom.2016.0410.

Kumjian, M. R., and A. V. Ryzhkov, 2008: Polarimetric signatures in supercell thunderstorms. J. Appl. Meteor. Climatol., 47, 1940-1961, https://doi.org/10.1175/2007JAMC1874.1.

_ and _ 2009: Storm-relative helicity revealed from polarimetric radar measurements. J. Atmos. Sci., 66, 667-685, https://doi.org/10.1175/2008JAS2815.1.

$\longrightarrow,-$, H. D. Reeves, and T. J. Schuur, 2013: A dualpolarization radar signature of hydrometeor refreezing in winter storms. J. Appl. Meteor. Climatol., 52, 2549-2566, https://doi.org/10.1175/JAMC-D-12-0311.1.

Kuster, C. M., P. L. Heinselman, J. C. Snyder, K. A. Wilson, D. A Speheger, and J. E. Hocker, 2017: An evaluation of radarbased tornado track estimation products by Oklahoma public safety officials. Wea. Forecasting, 32, 1711-1726, https:// doi.org/10.1175/WAF-D-17-0031.1.

Lakshmanan, V., A. Fritz, T. Smith, K. Hondl, and G. Stumpf, 2007a: An automated technique to quality control radar reflectivity data. J. Appl. Meteor. Climatol., 46, 288-305, https:// doi.org/10.1175/JAM2460.1.

- T. Smith, G. J. Stumpf, and K. Hondl, 2007b: The Warning Decision Support System-Integrated Information. Wea Forecasting, 22, 596-612, https://doi.org/10.1175/WAF1009.1. 
Manross, K. L., T. M. Smith, J. T. Ferree, and G. J. Stumpf, 2008: An on-demand user interface for requesting multi-radar, multi-sensor time accumulated products to support severe weather verification. 24th Conf. on Interactive Information Processing Systems, New Orleans, LA, Amer. Meteor. Soc., P2.13, https://ams.confex.com/ams/88Annual/techprogram/ paper_134621.htm.

Markowski, P. M., 2002: Hook echoes and rear-flank downdrafts: A review. Mon. Wea. Rev., 130, 852-876, https://doi.org/10.1175/ 1520-0493(2002)130<0852:HEARFD > 2.0.CO;2.

Miller, M. L., V. Lakshmanan, and T. M. Smith, 2013: An automated method for depicting mesocyclone paths and intensities. Wea. Forecasting, 28, 570-585, https://doi.org/10.1175/ WAF-D-12-00065.1.

Mitchell, E. D., S. V. Vasiloff, G. J. Stumpf, A. Witt, M. D. Eilts, J. T. Johnson, and K. W. Thomas, 1998: The National Severe Storms Laboratory tornado detection algorithm. Wea. Forecasting, 13, 352-366, https://doi.org/10.1175/1520-0434(1998) $013<0352$ :TNSSLT $>2.0$. CO 2 .

Newman, J. F., V. Lakshmanan, P. L. Heinselman, M. B. Richman, and T. M. Smith, 2013: Range-correcting azimuthal shear in Doppler radar data. Wea. Forecasting, 28, 194-211, https:// doi.org/10.1175/WAF-D-11-00154.1.

Ortega, K. L., J. M. Krause, and A. V. Ryzhkov, 2016: Polarimetric radar characteristics of melting hail. Part III: Validation of the algorithm for hail size discrimination. J. Appl. Meteor. Climatol., 55, 829-848, https://doi.org/10.1175/JAMC-D-15-0203.1.

_ , D. W. Burgess, K. L. Elmore, M. C. Mahalik, and B. R. Smith, 2018: Severe weather algorithm development and improvement activities for the WSR-88D at NSSL/CIMMS under the ROC tech transfer MOU. 29th Conf. on Weather Analysis and Forecasting, Denver, CO, Amer. Meteor. Soc., 12A.4, https://ams.confex.com/ams/29WAF25NWP/webprogram/ Paper344948.html.

Park, H.-S., A. V. Ryzhkov, D. S. Zrnić, and K.-E. Kim, 2009: The hydrometeor classification algorithm for the polarimetric WSR88D: Description and application to an MCS. Wea. Forecasting, 24, 730-748, https://doi.org/10.1175/2008WAF2222205.1.

Pazmany, A. L., J. B. Mead, H. B. Bluestein, J. C. Snyder, and J. B. Houser, 2013: A mobile, rapid-scanning, X-band, polarimetric (RaXPol) Doppler radar system. J. Atmos. Oceanic Technol., 30, 1398-1413, https://doi.org/10.1175/JTECH-D-12-00166.1.

Piltz, S. F., and D. W. Burgess, 2009: The impacts of thunderstorm geometry and WSR-88D beam characteristics on diagnosing supercell tornadoes. 34th Conf. on Radar Meteorology, Williamsburg, VA, Amer. Meteor. Soc., P6.18, https://ams. confex.com/ams/34Radar/techprogram/paper_155944.htm.

Przybylinski, R. W., 1995: The bow echo: Observations, numerical simulations, and severe weather detections. Wea. Forecasting, 10, 203-218, https://doi.org/10.1175/1520-0434(1995)010<0203: TBEONS $>2.0 . \mathrm{CO} ; 2$.

Roberts, R. D., and J. W. Wilson, 1989: A proposed microburst nowcasting procedure using single-Doppler radar. J. Appl. Meteor. Climatol., 28, 285-303, https://doi.org/10.1175/15200450(1989)028<0285:APMNPU>2.0.CO;2.

Ryzhkov, A. V., and D. S. Zrnić, 1998: Discrimination between rain and snow with a polarimetric radar. J. Appl. Meteor., 37, 1228-1240, https://doi.org/10.1175/1520-0450(1998)037<1228: DBRASW $>2.0 . \mathrm{CO} ; 2$

, - - and B. A. Gordon, 1998: Polarimetric method for ice water content determination. J. Appl. Meteor., 37, 125-134, https://doi.org/10.1175/1520-0450(1998)037<0125:PMFIWC> 2.0.CO;2.
— T. J. Schuur, D. W. Burgess, and D. S. Zrnić, 2005: Polarimetric tornado detection. J. Appl. Meteor. Climatol., 44, 557570, https://doi.org/10.1175/JAM2235.1.

Schmidhuber, J., 2015: Deep learning in neural networks: An overview. Neural Networks, 61, 85-117, https://doi.org/ 10.1016/j.neunet.2014.09.003.

Schmocker, G. K., R. W. Przybylinski, and Y. J. Lin, 1996: Forecasting the initial onset of damaging downburst winds associated with a Mesoscale Convective System (MCS) using the Mid-Altitude Radial Convergence (MARC) signature. Preprints, 15th Conf. on Weather Analysis and Forecasting, Norfolk, VA, Amer. Meteor. Soc., 306-311.

Skinner, P. S., L. J. Wicker, D. M. Wheatley, and K. H. Knopfmeier, 2016: Application of two spatial verification methods to ensemble forecasts of low-level rotation. Wea. Forecasting, 31, 713-735, https://doi.org/10.1175/WAF-D-15-0129.1.

Smith, B. T., R. L. Thompson, A. R. Dean, and P. T. Marsh, 2015: Diagnosing the conditional probability of tornado damage rating using environmental and radar attributes. Wea. Forecasting, 30, 914-932, https://doi.org/10.1175/WAF-D-14-00122.1.

Smith, T. M., and K. L. Elmore, 2004: The use of radial velocity derivatives to diagnose rotation and divergence. Preprints, 11th Conf. on Aviation, Range, and Aerospace, Hyannis, MA, Amer. Meteor. Soc., P5.6, http://ams.confex.com/ams/pdfpapers/ 81827.pdf.

- - - and S. A. Dulin, 2004: A damaging downburst prediction and detection algorithm for the WSR-88D. Wea. Forecasting, 19, 240-250, https://doi.org/10.1175/1520-0434(2004) 019<0240:ADDPAD>2.0.CO;2.

, and Coauthors, 2016: Multi-Radar Multi-Sensor (MRMS) severe weather and aviation products: Initial operating capabilities. Bull. Amer. Meteor. Soc., 97, 1617-1630, https:// doi.org/10.1175/BAMS-D-14-00173.1.

Snyder, J. C., and A. V. Ryzhkov, 2015: Automated detection of polarimetric tornadic debris signatures using a hydrometeor classification algorithm. J. Appl. Meteor. Climatol., 54, 18611870, https://doi.org/10.1175/JAMC-D-15-0138.1.

, — - H. B. Bluestein, and S. F. Blair, 2014: Polarimetric analysis of two giant-hail producing supercells by X-band and S-band radars. 27th Conf. on Severe Local Storms, Madison, WI, Amer. Meteor. Soc., 166, https://ams.confex.com/ams/ 27SLS/webprogram/Paper255455.html.

- _- J. Jrause, and C. Kuster, 2017: Identifying polarimetric radar signatures aloft associated with large and giant hail. 38th Conf. on Radar Meteorology, Chicago, IL, Amer. Meteor. Soc., 237, https://ams.confex.com/ams/38RADAR/meetingapp.cgi/ Paper/321184.

Spratt, S. M., D. W. Sharp, P. Welsh, A. Sandrik, F. Alsheimer, and C. Paxton, 1997: A WSR-88D assessment of tropical cyclone outer rainband tornadoes. Wea. Forecasting, 12, 479-501, https://doi.org/10.1175/1520-0434(1997)012<0479: AWAOTC $>2.0 . \mathrm{CO} ; 2$.

Stough, S. M., L. D. Carey, C. J. Schultz, and P. M. Bitzer, 2017: Investigating the relationship between lightning and mesocyclonic rotation in supercell thunderstorms. Wea. Forecasting, 32, 2237-2259, https://doi.org/10.1175/WAFD-17-0025.1.

Stumpf, G. J., A. Witt, E. D. Mitchell, P. L. Spencer, J. T. Johnson, M. D. Eilts, K. W. Thomas, and D. W. Burgess, 1998: The National Severe Storms Laboratory mesocyclone detection algorithm for the WSR-88D. Wea. Forecasting, 13, 304-326, https://doi.org/10.1175/1520-0434(1998)013<0304: TNSSLM $>2.0 . \mathrm{CO} ; 2$. 
and Coauthors, 2012: The Experimental Warning Program's 2011 and 2012 Spring Experiments at the NOAA Hazardous Weather Testbed. 26th Conf. on Severe Local Storms, Nashville, TN, Amer. Meteor. Soc., 3.4, https://ams.confex.com/ams/26SLS/ webprogram/Paper212324.html.

Thompson, R. L., and Coauthors, 2017: Tornado damage rating probabilities derived from WSR-88D data. Wea. Forecasting, 32, 1509-1528, https://doi.org/10.1175/WAF-D-17-0004.1.

Torres, S. M., and C. D. Curtis, 2007: Initial implementation of super-resolution data on the NEXRAD network. 23rd Conf. on Information Processing Systems, San Antonio, TX, Amer. Meteor. Soc., 5B.10, https://ams.confex.com/ams/ 87ANNUAL/techprogram/paper_116240.htm.

Wienhoff, Z. B., H. B. Bluestein, L. J. Wicker, J. C. Snyder, A. Shapiro, C. K. Potvin, and D. W. Reif, 2017: An analysis of the evolution and structure of a multiple-tornado-producing supercell near Dodge City, KS, on 24 May 2016. 38th Conf. on Radar Meteorology, Chicago, IL, Amer. Meteor. Soc., 147, https://ams.confex.com/ams/38RADAR/meetingapp.cgi/Paper/ 320832 .

Wilson, J. W., G. B. Foote, N. A. Crook, J. C. Fankhouser, C. G. Wade, J. D. Tuttle, C. K. Mueller, and S. K. Krueger, 1992: The role of boundary-layer convergence zones and horizontal rolls in the initiation of thunderstorms: A case study. Mon. Wea. Rev., 120, 1785-1815, https://doi.org/10.1175/1520-0493(1992) $120<1785$ :TROBLC $>2.0$. CO; 2 .
Witt, A., M. D. Eilts, G. J. Stumpf, J. T. Johnson, E. D. Mitchell, and K. W. Thomas, 1998: An enhanced hail detection algorithm for the WSR-88D. Wea. Forecasting, 13, 286-303, https:// doi.org/10.1175/1520-0434(1998)013<0286:AEHDAF>2.0.CO;2.

_ R. R. A. Brown, and Z. Jing, 2009: Performance of a new velocity dealiasing algorithm for the WSR-88D. 34th Conf. on Radar Meteorology, Williamsburg, VA, Amer. Meteor. Soc., P4.8, https:// ams.confex.com/ams/34Radar/techprogram/paper_155951.htm.

Wood, V. T., and R. A. Brown, 1997: Effects of radar sampling on single-Doppler velocity signatures of mesocyclones and tornadoes. Wea. Forecasting, 12, 928-938, https://doi.org/10.1175/ 1520-0434(1997)012<0928:EORSOS>2.0.CO;2.

$\longrightarrow$, and -2011 : Simulate tornadic vortex signatures of tornado-like vortices having one- and two-celled structures J. Appl. Meteor. Climatol., 50, 2338-2342, https://doi.org/ 10.1175/JAMC-D-11-0118.1.

Zittel, W. D., R. R. Lee, E. D. Mitchell, and D. Sirmans, 2001: Environmental and signal processing conditions that negatively impact the performance of the WSR-88D tornado detection algorithm. 30th Int. Conf. on Radar, Munich Germany, Amer. Meteor. Soc., 12B.6, https://ams.confex.com/ams/ pdfpapers/19862.pdf.

Zrnić, D. S., and R. J. Doviak, 1975: Velocity spectra of vortices scanned with a pulse-Doppler radar. J. Appl. Meteor., 14, 1531-1539, https://doi.org/10.1175/1520-0450(1975)014<1531: VSOVSW $>2.0 . \mathrm{CO} ; 2$. 


\title{
CORRIGENDA
}

\section{Corrigendum}

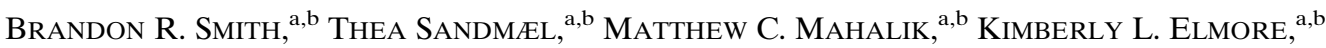 \\ Darrel M. Kingfield, ${ }^{\mathrm{a}, \mathrm{b}}$ Kiel L. OrTega, ${ }^{\mathrm{a}, \mathrm{b}}$ AND Travis M. SMith ${ }^{\mathrm{a}, \mathrm{b}}$ \\ ${ }^{a}$ Cooperative Institute for Mesoscale Meteorological Studies, University of Oklahoma, Norman, Oklahoma \\ ${ }^{\mathrm{b}}$ NOAA/OAR National Severe Storms Laboratory, Norman, Oklahoma
}

(Manuscript received 21 July 2020, in final form 22 March 2021)

The purpose of this corrigendum is to address errors in several equations that were discovered in Mahalik et al. (2019, hereafter M19), which presented the derivations for both the azimuthal and divergent shear linear least squares derivative (LLSD) equations that serve as the foundation for the publication. While two of the errors are minor and typographical in nature, additional errors in two of the matrices, the coefficient matrix [M19's Eq. (8)] and the adjugate matrix [M19's Eq. (10)], introduce incorrect components within the final versions of the LLSD horizontal shear equations provided in M19's Eqs. (12a) and (12b), in addition to their fully expanded forms in M19's appendixes A and B. The errors and their subsequent corrections are addressed below in the order that they are encountered within M19.

The first two equation errors in M19 are both typographical in nature. The $\Delta$ (delta) modifier was incorrectly excluded from the range component of the two-dimensional radar variable $u_{i j}$, shown in M19's Eq. (5). This delta is needed as it describes the offset in range from the center of the kernel in which the derivatives are being calculated. The updated M19's Eq. (5) is as follows:

$$
R=\sum_{k=0}^{m \times n} w_{k}\left[u\left(\Delta r_{k}, \Delta \theta_{k}\right)-\left(u_{0}+u_{r} \Delta r_{k}+u_{\theta} \Delta \theta_{k}\right)\right]^{2} .
$$

Similarly, the $\Delta$ (delta) modifier was incorrectly excluded from the azimuth offset in M19's Eq. (6c):

$$
\frac{\partial R}{\partial u_{0}}=0=\sum_{k=0}^{m \times n} 2\left(-u_{k}+u_{0}+u_{r} \Delta r_{k}+u_{\theta} \Delta \theta_{k}\right) w_{k} .
$$

The remainder of the equation errors are attributed to an incorrect setup of both the coefficient matrix used to express the complete system of LLSD equations and its associated adjugate matrix. M19's Eq. (8), which outlines the complete system of LLSD equations expressed in matrix form, contains three matrices, the details of which are outlined in M19. Out of the three matrices, only the coefficient matrix contains errors. These errors relate only to the cross-diagonal coefficients, which are incorrectly switched across the diagonal, not allowing the original LLSD equations in M19's Eqs. (7a)-(7c) to be generated when the coefficient and variable matrices are multiplied. To correct this, these cross-diagonal coefficients are held in their original element positions, producing the corrected M19's Eq. (8) below:

$$
\left[\begin{array}{ccc}
\Sigma w_{k} \Delta r_{k} \Delta \theta_{k} & \Sigma w_{k} \Delta r_{k}^{2} & \Sigma w_{k} \Delta r_{k} \\
\Sigma w_{k} \Delta \theta_{k}^{2} & \Sigma w_{k} \Delta r_{k} \Delta \theta_{k} & \Sigma w_{k} \Delta \theta_{k} \\
\Sigma w_{k} \Delta \theta_{k} & \Sigma w_{k} \Delta r_{k} & \Sigma w_{k}
\end{array}\right]\left[\begin{array}{c}
u_{\theta} \\
u_{r} \\
u_{0}
\end{array}\right]=\left[\begin{array}{c}
\Sigma w_{k} \Delta r_{k} u_{k} \\
\Sigma w_{k} \Delta \theta_{k} u_{k} \\
\Sigma w_{k} u_{k}
\end{array}\right] .
$$

Mahalik's current affiliation: Cherokee Nation Strategic Programs and NOAA/OAR Weather Program Office, Silver Spring, Maryland.

Kingfield's current affiliation: Cooperative Institute for Research in Environmental Sciences, University of Colorado Boulder, and NOAA/OAR/ESRL/Global Systems Laboratory, Boulder, Colorado.

Corresponding author: Brandon R. Smith, brandon.r.smith@noaa.gov

DOI: 10.1175/WAF-D-20-0125.1

(c) 2021 American Meteorological Society. For information regarding reuse of this content and general copyright information, consult the AMS Copyright Policy (www.ametsoc.org/PUBSReuseLicenses). 
As discussed in M19 and shown by M19's Eq. (9), the derivatives of the individual LLSD azimuthal and divergent shear components are found by multiplying the answer matrix by the inverse of the coefficient matrix. This inverse is found by calculating both the determinate and adjugate of the coefficient matrix. The adjugate matrix shown in M19's Eq. (10), however, is not set up correctly. Part of the process of creating the adjugate matrix involves switching the cross-diagonal elements. In the case of M19's Eq. (10), only the $a_{23}$ and $a_{32}$ elements were switched. To correctly create the adjugate matrix, the $a_{12}$ element must be switched with the $a_{21}$ and the $a_{13}$ element switched with the $a_{31}$. Adjusting M19's Eq. (10) to account for these errors produces the following corrected equation:

$$
\mathbf{M}^{-1}=\frac{1}{D} \operatorname{adj}(\mathbf{M})=\left[\begin{array}{lll}
a_{11} & a_{21} & a_{31} \\
a_{12} & a_{22} & a_{32} \\
a_{13} & a_{23} & a_{33}
\end{array}\right]\left(\frac{1}{D}\right) .
$$

Since the adjugate matrix shown in M19's Eq. (10) had to be corrected, all downstream equations have been adjusted using the corrected adjugate matrix. The corrected versions of these downstream equations are displayed below where Eq. (5) is the corrected form of M19's Eq. (11), Eq. (6) is the corrected form of M19's Eq. (12a), and Eq. (7) is the corrected form of M19's Eq. (12b):

$$
\begin{aligned}
{\left[\begin{array}{l}
u_{\theta} \\
u_{r} \\
u_{0}
\end{array}\right] } & =\left[\begin{array}{lll}
a_{11} / D & a_{21} / D & a_{31} / D \\
a_{12} / D & a_{22} / D & a_{32} / D \\
a_{13} / D & a_{23} / D & a_{33} / D
\end{array}\right]\left[\begin{array}{c}
\Sigma w_{k} \Delta r_{k} u_{k} \\
\Sigma w_{k} \Delta \theta_{k} u_{k} \\
\Sigma w_{k} u_{k}
\end{array}\right], \\
u_{\theta} & =\sum_{k=0}^{m \times n} w_{k} \Delta r_{k} u_{k}\left(\frac{a_{11}}{D}\right)+\sum_{k=0}^{m \times n} w_{k} \Delta \theta_{k} u_{k}\left(\frac{a_{21}}{D}\right)+\sum_{k=0}^{m \times n} w_{k} u_{k}\left(\frac{a_{31}}{D}\right), \\
u_{r} & =\sum_{k=0}^{m \times n} w_{k} \Delta r_{k} u_{k}\left(\frac{a_{12}}{D}\right)+\sum_{k=0}^{m \times n} w_{k} \Delta \theta_{k} u_{k}\left(\frac{a_{22}}{D}\right)+\sum_{k=0}^{m \times n} w_{k} u_{k}\left(\frac{a_{32}}{D}\right) .
\end{aligned}
$$

The corrected versions of M19's appendixes A and B, the expanded forms of M19's Eqs. (12a) and (12b) [Eqs. (6) and (7) above, respectively] are shown, respectively, in Eqs. (8) and (9) below. Using Eq. (3), the original determinate in M19's appendixes A and B was also corrected and is also shown in Eqs. (8) and (9):

$$
\begin{aligned}
u_{\theta}= & \left(\sum_{k=0}^{m \times n} \Delta r_{k} \Delta \theta_{k} \sum_{k=0}^{m \times n} \Delta r_{k} \Delta \theta_{k} \sum_{k=0}^{m \times n} w_{k}-2 \sum_{k=0}^{m \times n} \Delta r_{k} \Delta \theta_{k} \sum_{k=0}^{m \times n} \Delta \theta_{k} \sum_{k=0}^{m \times n} \Delta r_{k}\right. \\
& \left.+\sum_{k=0}^{m \times n} \Delta r_{k}^{2} \sum_{k=0}^{m \times n} \Delta \theta_{k} \sum_{k=0}^{m \times n} \Delta \theta_{k}-\sum_{k=0}^{m \times n} \Delta r_{k}^{2} \sum_{k=0}^{m \times n} \Delta \theta_{k}^{2} \sum_{k=0}^{m \times n} w_{k}+\sum_{k=0}^{m \times n} \Delta r_{k} \sum_{k=0}^{m \times n} \Delta \theta_{k}^{2} \sum_{k=0}^{m \times n} \Delta r_{k}\right)^{-1} \\
& {\left[\sum_{k=0}^{m \times n} \Delta r_{k} u_{k}\left(\sum_{k=0}^{m \times n} \Delta r_{k} \Delta \theta_{k} \sum_{k=0}^{m \times n} w_{k}-\sum_{k=0}^{m \times n} \Delta \theta_{k} \sum_{k=0}^{m \times n} \Delta r_{k}\right)\right.} \\
& +\sum_{k=0}^{m \times n} \Delta \theta_{k} u_{k}\left(\sum_{k=0}^{m \times n} \Delta r_{k} \sum_{k=0}^{m \times n} \Delta r_{k}-\sum_{k=0}^{m \times n} r_{k}^{2} \sum_{k=0}^{m \times n} w_{k}\right) \\
& \left.+\sum_{k=0}^{m \times n} u_{k}\left(\sum_{k=0}^{m \times n} \Delta r_{k}^{2} \sum_{k=0}^{m \times n} \Delta \theta_{k}-\sum_{k=0}^{m \times n} \Delta r_{k} \sum_{k=0}^{m \times n} \Delta r_{k} \Delta \theta_{k}\right)\right], \\
u_{r}= & \left(\sum_{k=0}^{m \times n} \Delta r_{k} \Delta \theta_{k} \sum_{k=0}^{m \times n} \Delta r_{k} \Delta \theta_{k} \sum_{k=0}^{m \times n} w_{k}-2 \sum_{k=0}^{m \times n} \Delta r_{k} \Delta \theta_{k} \sum_{k=0}^{m \times n} \Delta \theta_{k} \sum_{k=0}^{m \times n} \Delta r_{k}\right. \\
& \left.+\sum_{k=0}^{m \times n} \Delta r_{k}^{2} \sum_{k=0}^{m \times n} \Delta \theta_{k} \sum_{k=0}^{m \times n} \Delta \theta_{k}-\sum_{k=0}^{m \times n} \Delta r_{k}^{2} \sum_{k=0}^{m \times n} \Delta \theta_{k}^{2} \sum_{k=0}^{m \times n} w_{k}+\sum_{k=0}^{m \times n} \Delta r_{k} \sum_{k=0}^{m \times n} \Delta \theta_{k}^{2} \sum_{k=0}^{m \times n} \Delta r_{k}\right)^{-1} \\
& {\left[\sum_{k=0}^{m \times n} \Delta r_{k} u_{k}\left(\sum_{k=0}^{m \times n} \Delta \theta_{k} \sum_{k=0}^{m \times n} \Delta \theta_{k}-\sum_{k=0}^{m \times n} \theta_{k}^{2} \sum_{k=0}^{m \times n} w_{k}\right)+\sum_{k=0}^{m \times n} \Delta \theta_{k} u_{k}\left(\sum_{k=0}^{m \times n} \Delta r_{k} \Delta \theta_{k} \sum_{k=0}^{m \times n} w_{k}\right.\right.} \\
& \left.\left.-\sum_{k=0}^{m \times n} \Delta r_{k} \sum_{k=0}^{m \times n} \Delta \theta_{k}\right)+\sum_{k=0}^{m \times n} u_{k}\left(\sum_{k=0}^{m \times n} \Delta r_{k} \sum_{k=0}^{m \times n} \theta_{k}^{2}-\sum_{k=0}^{m \times n} \Delta r_{k} \Delta \theta_{k} \sum_{k=0}^{m \times n} \Delta \theta_{k}\right)\right] .
\end{aligned}
$$

It should be noted that while M19 did not include the correct final version of the LLSD azimuthal shear (AzShear) equation, the correct version is employed in the operational code set that is utilized in the workflow to generate the rotation track products within the current Multi-Radar Multi-Sensor (MRMS) system. In addition, the figures in M19 are unaffected by these equation errors. 
Acknowledgments. We thank Monika Freeman, with the Environmental Remote Sensing Laboratory at École Polytechnique Férédale de Lausanne and the Radar, Satellite, and Nowcasting Division at MeteoSwiss, who discovered the errors and notified the authors while providing documentation of the work that had been done to uncover the errors. Monika's documentation helped immensely in the diagnosis and subsequent correction of the incorrect equations. Funding was provided by NOAA/ Office of Oceanic and Atmospheric Research under NOAA-University of Oklahoma Cooperative Agreement NA16OAR4320115, U.S. Department of Commerce.

\section{REFERENCE}

Mahalik, M. C., B. R. Smith, K. L. Elmore, D. M. Kingfield, K. L. Ortega, and T. M. Smith, 2019: Estimates of gradients in radar moments using a linear least squares derivative technique. Wea. Forecasting, 34, 415434, https://doi.org/10.1175/WAF-D-18-0095.1. 